\title{
Gas permeation properties of thermally rearranged (TR) isomers and their aromatic polyimide precursors
}

\author{
Qiang Liu ${ }^{1}$, Hailun Borjigin ${ }^{2}$, Donald R. Paul ${ }^{1}$, J. S. Riffle ${ }^{2}$, \\ James E. McGrath ${ }^{2}$, and Benny D. Freeman ${ }^{1 *}$
}

1. Department of Chemical Engineering, Texas Materials Institute, Center for Energy and

Environmental Research, The University of Texas at Austin, 10100 Burnet Road, Bldg. 133, Austin, TX 78758, USA

2. Department of Chemistry, Macromolecules and Interfaces Institute, Virginia Tech, Blacksburg, VA 24061, USA

Submission to Journal of Membrane Science

*Corresponding Author: Email freeman@che.utexas.edu Tel: 1-512-232-2803 
Graphical Abstract

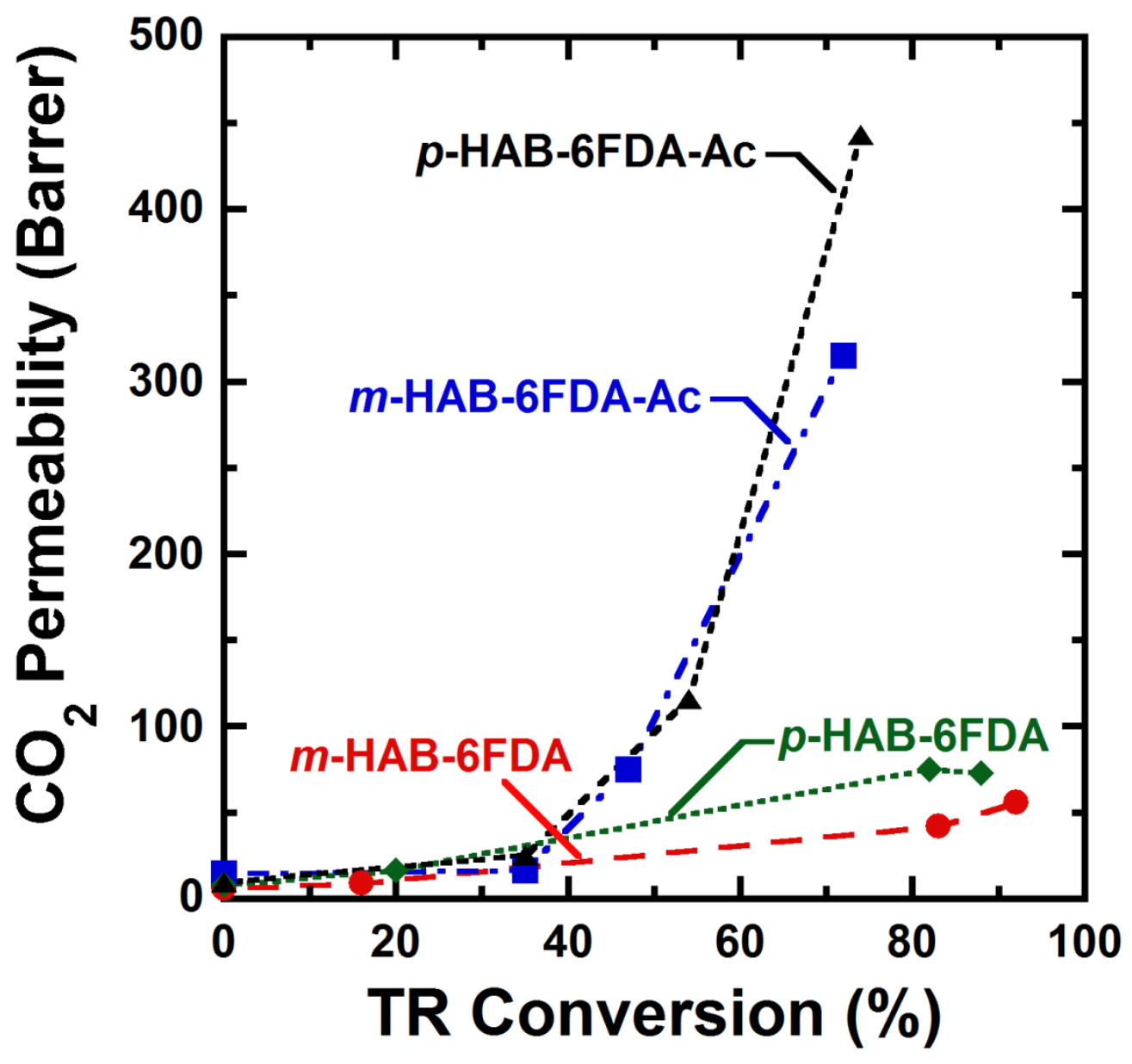




\begin{abstract}
This study explores the influence of metalpara and ortho-position functional group structures on gas transport properties of two isomeric polyimides and their thermally rearranged (TR) polymers. The diamine isomers, 3,3'-dihydroxy-4,4'-diamino-biphenyl ( $p$-HAB) and 4,4'dihydroxybiphenyl-3,3'-diamino-biphenyl $(m$-HAB), were polymerized with 2,2'-bis-(3,4dicarboxy-phenyl) hexafluoropropane dianhydride (6FDA) to form two aromatic polyimide isomers. These polyimides were also prepared with either hydroxyl or acetate groups at the ortho-positions to the imide ring, and the polyimides were partially converted to the corresponding polybenzoxazoles by thermal treatment at various temperatures and times. Single gas permeabilities of $\mathrm{CH}_{4}, \mathrm{H}_{2}, \mathrm{O}_{2}, \mathrm{~N}_{2}$ and $\mathrm{CO}_{2}$ were measured at $35^{\circ} \mathrm{C}$ and upstream pressures ranging from $3 \mathrm{~atm}$ to $17 \mathrm{~atm}$. Polyacetylimides and their corresponding TR polymers showed higher gas permeability and lower selectivity than polyhydroxyimides and their TR analogs, respectively. The effect of isomerism on gas transport properties was also investigated. Paraconnected polyhydroxyimide had higher fractional free volume, and thus, gas permeability coefficients than meta-connected polyhydroxyimide. Interestingly, in contrast to the meta/para effects previously reported in the literature for linear aromatic polymers, the meta-linked polyacetylimide had higher permeabilities and lower selectivities than its para-linked analog, presumably due to steric hindrance from the acetate groups that may inhibit the rotational mobility of the phenylene units in the para-linked isomer. For TR polymers derived from either polyhydroxyimides or polyacetylimides, para-configured polymers had higher gas permeabilities than meta-configured analogs. Finally, polyimide precursors for TR polymers can be sensitive to synthesis and casting conditions, and this study demonstrates how small variations in synthesis and casting procedure can have a significant effect on physical and gas permeation properties.
\end{abstract}


Keyword: Thermally rearranged; isomer; polybenzoxazole; membrane 


\section{Introduction}

Membrane technology for gas separation has grown into a business with system sales approaching $\$ 1$ billion [1]. Today, a major application in membrane purification is natural gas sweetening, where, in the U.S., $\mathrm{CO}_{2}$ must be reduced to levels below $2 \%$ to meet pipeline specifications [1-3]. Thermally rearranged (TR) polymers, first investigated by Park et al. [4], have shown promising gas transport properties, including for $\mathrm{CO}_{2} / \mathrm{CH}_{4}$ separation. TR polymers can be polybenzoxazoles (PBO), polybenzimidazoles (PBI), or polybenzothiazoles (PBT) composed of rigid-rod structures with high-torsional energy barriers to rotation between phenylene-heterocyclic rings [4-6]. Because of their insolubility in common organic solvents, many polybenzoxazoles cannot be solution-cast as membranes. Tullos et al. overcame this challenge by preparing ortho-functional polyhydroxyimides (o-PHIs) that readily dissolved in common solvents and then converted them to insoluble polybenzoxazoles by thermal treatment [6]. In 2007, Park et al. extended this idea to gas separation membranes [4]. Since this initial report, much research has focused on this interesting family of materials [7-21].

Recent studies have investigated the influence of polyimide precursor structure on properties of TR polymers. For example, Calle et al. identified a correlation between the polyimide glass transition temperature, $T_{g}$, and the thermal rearrangement onset temperature by studying 15 sets of $o$-PHIs and copolyimides derived from 6 diamines and 4 dianhydrides [7]. As the polyimide precursor $\mathrm{T}_{\mathrm{g}}$ decreased from $358{ }^{\circ} \mathrm{C}$ to $234{ }^{\circ} \mathrm{C}$, the onset temperature of thermal rearrangement decreased from $373{ }^{\circ} \mathrm{C}$ to $290{ }^{\circ} \mathrm{C}$ [7]. Guo et al. explored the effect of lowering $\mathrm{T}_{\mathrm{g}}$ on gas transport properties. By using a bisphenol A type dianhydride, for example, both $\mathrm{T}_{\mathrm{g}}$ and onset temperature of thermal rearrangement were reduced by approximately $100{ }^{\circ} \mathrm{C}$. However, upon thermal rearrangement, gas permeabilities of these TR polymers were about five 
times lower than previously reported TR materials prepared at higher rearrangement temperatures $[11,18]$. Scholes et al. studied poly(benzoxazole-co-imide) membranes, which consist of TR-able $o$-PHI precursors and non-TR-able imide segments, and found that gas permeability and permselectivity could be tuned by varying the PBO to PI segment ratio [22]. Sanders et al. investigated the influence of various polyimide ortho-position functional groups on gas transport properties. As the ortho-position function group size increased, free volume and gas permeability of the polyimide precursors increased $[16,17]$. For example, $\mathrm{CO}_{2}$ permeability for a polyimide with a pivalate group was 18 times higher than that of a polyimide with a hydroxyl group at the ortho-position [17]. Furthermore, TR polymers formed from polyimides with non-hydroxyl ortho-position functional groups showed higher single gas permeabilities and lower selectivities than those formed from polyimides with hydroxyl groups [17].

This study focuses on the influence of metalpara orientation of two TR isomers on gas transport properties. Typically, for polymers with aromatic backbones, changing the aromatic ring connectivity from para to meta lowers $\mathrm{T}_{\mathrm{g}}$ and fractional free volume (FFV), resulting in decreases in gas solubility, diffusivity and, hence, permeability [23-27]. This effect has been reported in polysulfones [23, 28], polyimides [25, 27, 29, 30], polyesters [31], poly(phenolphthalein phthalates) [32], and polybenzimidazoles [33]. The meta/para effect has also been studied by molecular modeling that confirmed the experimental observations $[34,35]$. In this regard, Table 1 summarizes literature data comparing physical, thermal, and gas transport properties of several linear polymers having either para- or meta-connected aromatic rings. Three examples, described below, highlight the bulk of the literature findings on this topic. Then, one recent study on TR polymers is noted, and it suggests trends that are different from those found in previous studies. 
Table 1. Physical, thermal, and gas transport properties of several linear polymers having either para- or meta- connected aromatic rings. Aromatic units that are at the site of exchange from para to meta configuration are highlighted.

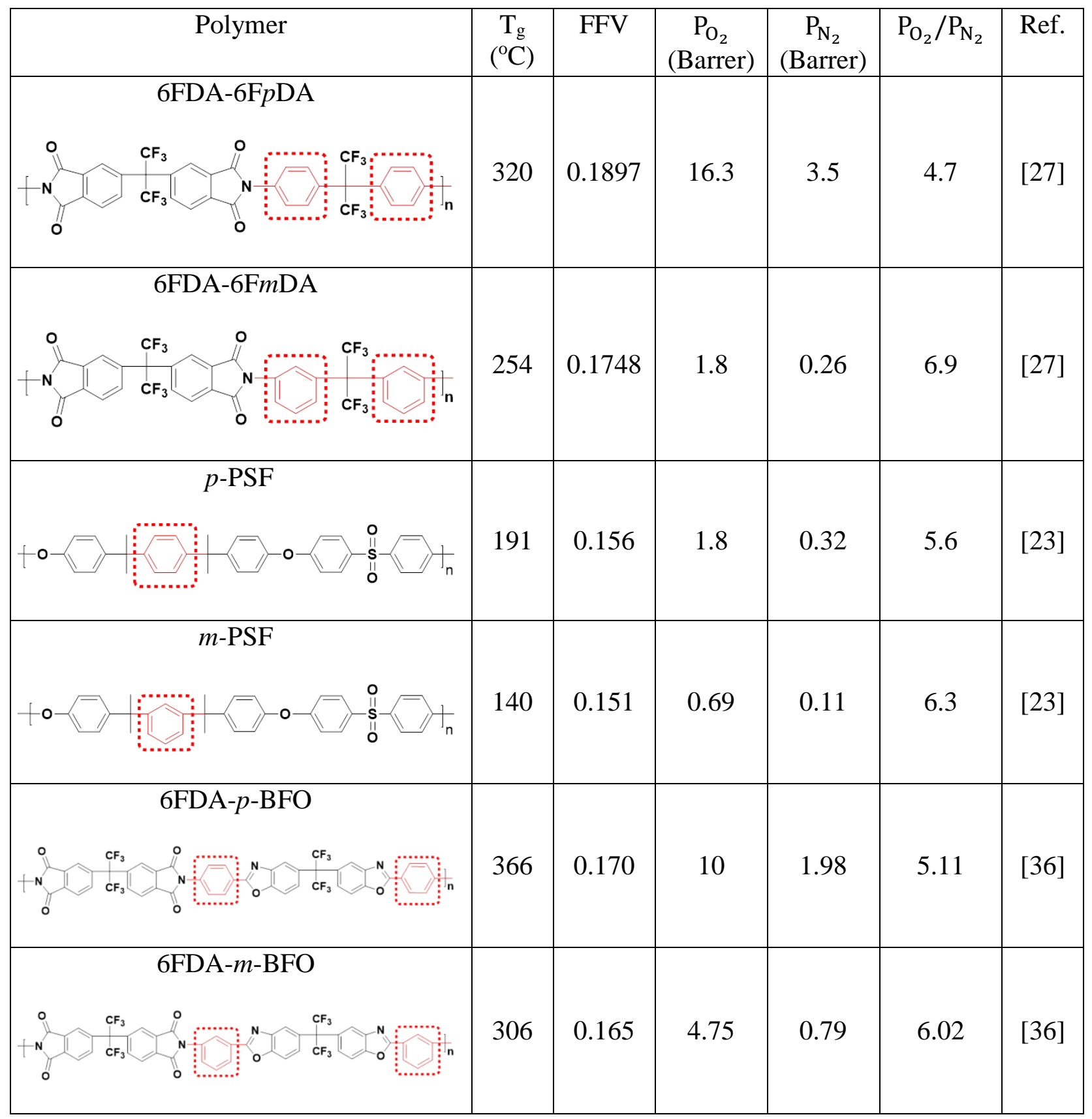

Coleman et al. explored the effect of metalpara structure on gas transport properties of 6FDA-6FDA polyimide $[25,27] . \mathrm{O}_{2}$ permeability of the para-connected 6FDA-6F $p$ DA was 
eight times higher than that of the meta-connected 6FDA-6FmDA, and the single gas $\mathrm{O}_{2} / \mathrm{N}_{2}$ selectivity of 6FDA-6FmDA was $47 \%$ higher than that of 6FDA-6FpDA. Coleman et al. also reported that the sub- $\mathrm{T}_{\mathrm{g}}$ transition for $6 \mathrm{FDA}-6 \mathrm{FpDA}$ occurred at a lower temperature and with higher intensity than for 6FDA-6FmDA. For polymers with linear aromatic backbones, such as polyimides and polysulfones, low-temperature peaks in dynamic mechanical spectra are often associated with phenyl ring rotations [23, 29]. Therefore, based on such changes observed in sub- $\mathrm{T}_{\mathrm{g}}$ relaxations, inter- and intra-segmental steric hindrance to phenylene ring rotation in 6FDA-6FmDA was believed to be more severe than in 6FDA-6FpDA. Apparently, the paraconnected phenylene groups could rotate freely, but the meta-connected phenylene units required a concerted motion of neighboring atoms for rotations to occur. As a result, the rotation freedom of para-linked 6FDA-6FpDA allowed higher fractional free volume (FFV), which led to higher gas permeability (cf., Table 1) [25, 27].

Aitken et al. conducted a similar study on polysulfones (PSF). As shown in Table 1, the $\mathrm{T}_{\mathrm{g}}$ of the meta isomer was $51{ }^{\circ} \mathrm{C}$ lower than that of the para isomer, indicating greater chain flexibility in meta-linked $m$-PSF than in para-linked $p$-PSF. In addition, Aitken et al. also reported a suppression in the sub- $\mathrm{T}_{\mathrm{g}}$ beta transition of $m$-PSF, which, as described earlier, signaled that $m$-PSF had more inhibition to rotational mobility of the phenylene groups than did the $p$-PSF. As a result, $p$-PSF, having higher local scale mobility than $m$-PSF despite the higher $\mathrm{T}_{\mathrm{g}}$ of $p$-PSF, had both higher FFV and higher gas permeability than $m$-PSF [23].

Recently Zhuang et al. investigated the effect of meta/para connectivity on physical properties and gas transport behavior of 6FDA-containing poly(benzoxazole-co-imide)s [36]. As presented in Table 1, para-linked 6FDA-p-BFO had higher gas permeability and lower selectivity than meta-linked 6FDA- $m$-BFO. For example, $\mathrm{O}_{2}$ permeability of 6FDA- $p$-BFO was 
about twice that of 6FDA-m-BFO, and single gas $\mathrm{O}_{2} / \mathrm{N}_{2}$ selectivity of 6 FDA- $p$-BFO was $18 \%$ lower than that of 6FDA- $m$-BFO. The $\mathrm{T}_{\mathrm{g}}$ of $6 \mathrm{FDA}-p-\mathrm{BFO}$ is $60^{\circ} \mathrm{C}$ higher than that of $m$-BPA. Zhuang et al. attributed the observed difference in gas permeability to higher diffusion coefficients in para-linked 6FDA-p-BFO relative to its meta-configured analog [36].

Comesaña-Gándara et al. explored the influence of metalpara orientation of two TR isomers on gas transport properties [37]. Interestingly, and in contrast to the general observations described above, the meta-connected polyhydroxyimide precursor displayed higher gas permeability than the para-connected polyhydroxyimide. Apparently, the meta-connected structure disrupted the polymer chain packing to a greater extent than the para-linked structure, as evident from the higher FFV for the meta-linked polyhydroxyimide [37]. As the polyhydroxyimides underwent thermal rearrangement, given the same extent of TR conversion, TR polymers derived from meta-connected polyhydroxyimide consistently showed higher gas permeability values than those derived from para-connected polyhydroxyimide [37]. However, as will be discussed later, a simple comparison of metalpara effects for partially rearranged TR polymers is difficult because a mixture of meta- and para-connected PBO rings could be obtained from thermal rearrangement of either meta- or para-linked polyimide precursors. Other factors, such as potential crosslinking of polymer chains during thermal rearrangement, can also make metalpara effects less predictable for TR polymers.

In this study, we investigate the effects of metalpara linkage and ortho-position functional group structure on single gas transport properties of two TR isomers to better understand these isomer effects in TR polymers. The two diamines considered, 4,4'-dihydroxy3,3'-diamino-biphenyl ( $m$-HAB) and 3,3'-dihydroxy-4,4'-diamino-biphenyl ( $p$-HAB), are isomers of one another. Polyhydroxylimides were prepared by polycondensation of one of the 
aforementioned diamines with 2,2'-bis-(3,4-dicarboxy-phenyl) hexafluoropropane dianhydride (6FDA). These polyhydroxyimides were also acetylated to form polyacetylimides, which are designated as $m$-HAB-6FDA-Ac (meta-linked) and $p$-HAB-6FDA-Ac (para-linked) in this paper. The motivation for this study is two-fold. First, previous studies have shown that gas transport properties of polyimide precursors and their TR analogs can depend on synthesis method [13]. Recently, we have discovered that casting solvent and conditions can also have a strong influence on permeation properties of these materials. A detailed study of casting solvent en gas transport properties of polyimide precursers and their TR analogs is currently under way in our laboratories and will be reported separately. Therefore, by studying polyhydroxyimides identical in structure but employing different synthesis and casting methods from those reported by Comesaña-Gándara et al., we wish to investigate whether the para/meta effects observed in this study still agree with those reported by Comesaña-Gándara et al. [37]. As mentioned earlier, gas transport properties of polyimide precursors and TR polymers can depend on the size of the ortho-position functional group. Because materials from earlier meta/para studies generally did not have any substituents on the aromatic rings that were being exchanged from meta to para configurations, this study seeks to explore whether a bulky substituent, in this case an acetate group, at the ortho-position may affect the metalpara relationship in polyimide precursors and their TR analogs.

The structures of the polyimide precursors and PBOs after thermal rearrangement are shown in Table 2. Although Table 2 shows the structure of the TR polymers as linear materials, it is likely that some interchain thermal rearrangement occurs, resulting in crosslinked structures $[4,18]$. Thermal properties, $\mathrm{T}_{\mathrm{g}}$, and fractional free volume of these materials are reported. In addition, single gas permeability and selectivity values of $\mathrm{CH}_{4}, \mathrm{~N}_{2}, \mathrm{O}_{2}, \mathrm{CO}_{2}$, and $\mathrm{H}_{2}$ are 
presented as a function of degree of TR conversion to help elucidate the role of metalpara orientation and ortho-position functional group structure on gas transport properties. 
Table 2. Chemical structures of polyimides considered in this study and their TR polymers.

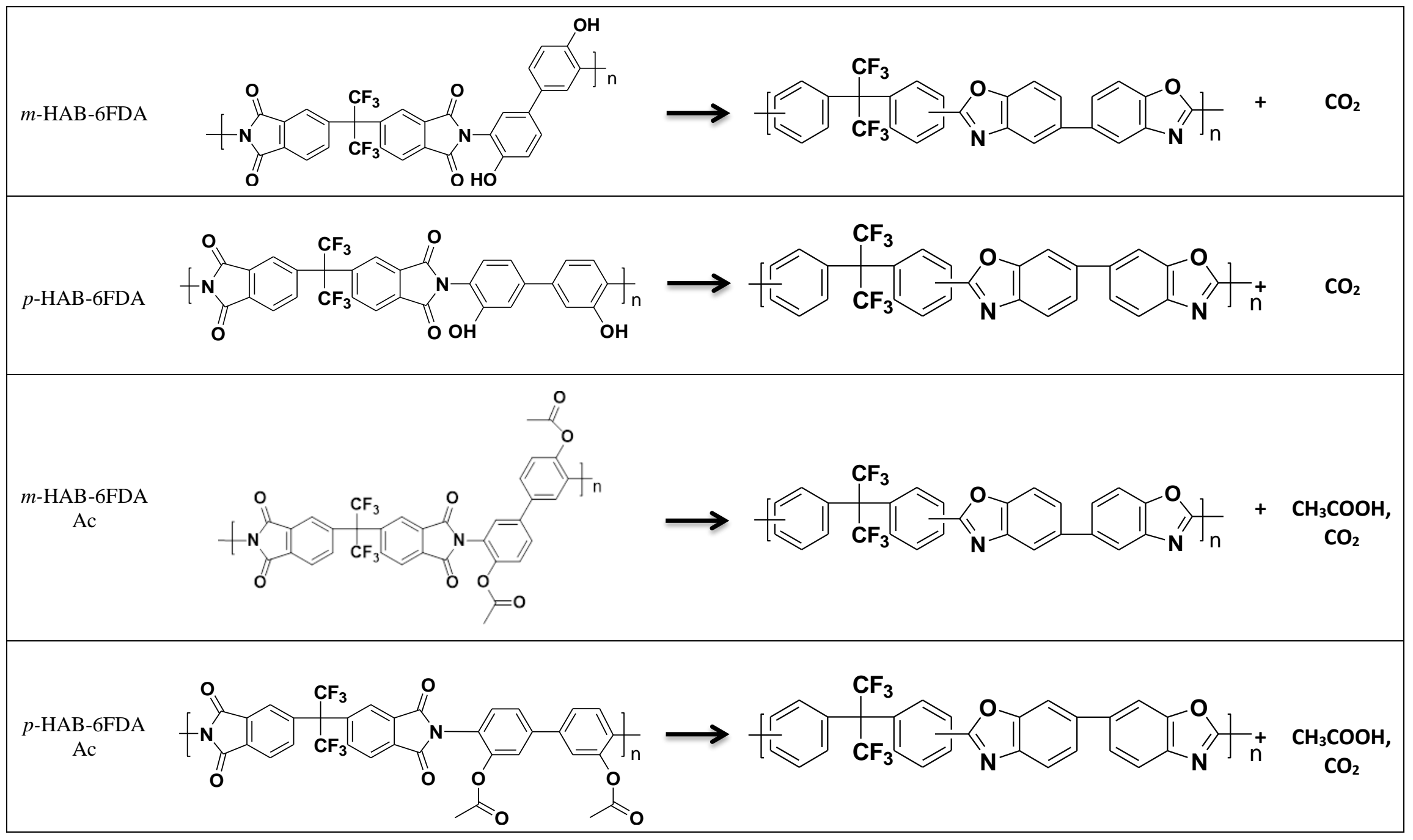




\section{Experimental}

\subsection{Polymer synthesis}

The diamine isomers used to synthesize the polyimide precursor were 3,3'-dihydroxy4,4'-diamino-biphenyl ( $p$-HAB) and 4,4'-dihydroxybiphenyl-3,3'-diamino-biphenyl ( $m$-HAB). The dianhydride was 2,2'-bis-(3,4-dicarboxy-phenyl) hexafluoropropane dianhydride (6FDA). $p$-HAB was purchased from Tokyo Chemical Industry (TCI) and used as received, and 6FDA was purchased from Alfa Aesar and dried in vacuum at $180{ }^{\circ} \mathrm{C}$ before use.

$m$-HAB was not commercially available and was synthesized as follows: Acetone (250 $\mathrm{ml})$ was added to 4,4'-biphenol $(12.2 \mathrm{~g}, 65.5 \mathrm{mmol})$ in a three-neck flask equipped with a condenser and mechanical stirrer. The mixture was heated to $60{ }^{\circ} \mathrm{C}$, and nitric acid $(7.9 \mathrm{ml}$ of $69.3 \%$ nitric acid, $131.0 \mathrm{mmol}$ ) was added dropwise. The solution was kept at $60{ }^{\circ} \mathrm{C}$ for $6 \mathrm{~h}$ and then washed with excess acetone. The product, 3,3'-dinitro-4,4'-dihydroxyl biphenyl (NHB), was filtered and dried under vacuum at $80{ }^{\circ} \mathrm{C}$ overnight (yield 87\%). NHB (11.6 g, $42.0 \mathrm{mmol}$ ) was then reacted with $1.1 \mathrm{~g} \mathrm{Pd} / \mathrm{C}$ in $200 \mathrm{ml}$ of NMP at $100{ }^{\circ} \mathrm{C}$. Hydrazine hydrate $(24 \mathrm{ml}, 290$ mmol) was then added dropwise. Afterwards, the solution was stirred and refluxed at $100{ }^{\circ} \mathrm{C}$ for $12 \mathrm{~h}$, and then hot-filtered through Celite. Methanol ( $200 \mathrm{~mL})$ was slowly added into the filtered solution. As the product cooled and crystallized, white crystals were collected and washed with deionized water. Finally, the product was dried in vacuum at $100{ }^{\circ} \mathrm{C}$ overnight. The yield was $81 \%$. The monomer degraded before melting.

Polyhydroxyimides were synthesized via an ester-acid method, as described previously $[10,38]$. For example, $7.0407 \mathrm{~g}$ of $6 \mathrm{FDA}(15.9 \mathrm{mmol})$ was reacted with $50 \mathrm{ml}$ of ethanol (856 $\mathrm{mmol})$ and $3 \mathrm{ml}$ of triethylamine $(21.5 \mathrm{mmol})$ in a three-neck flask equipped with a Dean-Stark 
trap, mechanical stirrer, and nitrogen purge. The mixture was heated to $90{ }^{\circ} \mathrm{C}$ and refluxed with stirring for $1 \mathrm{~h}$. Once the solution became clear, the trap was drained and an equimolar amount of $p$-HAB or $m$ - HAB $(3.4268 \mathrm{~g}, 15.9 \mathrm{mmol})$ was added, along with an $\sim 4 / 1(\mathrm{v} / \mathrm{v})$ ratio of $35 \mathrm{~mL}$ of NMP and $9 \mathrm{~mL}$ of $o$-dichlorobenzene as the azeotroping agent to produce a solids content of $\sim 30 \%(\mathrm{wt} / \mathrm{v})$. The mixture was then heated to $180{ }^{\circ} \mathrm{C}$ for $10 \mathrm{~h}$, precipitated in methanol, and dried under vacuum at $180{ }^{\circ} \mathrm{C}$ for $12 \mathrm{~h}$. The yield was $98 \%$.

To obtain polyacetylimides (Ac), 4.00 grams of polyhydroxyimide (6.4 hydroxyl eq) were dissolved in $20 \mathrm{ml}$ of NMP in a three-neck flask equipped with mechanical stirrer, condenser, and nitrogen purge. Then, $3.63 \mathrm{ml}$ of acetic anhydride $(38.4 \mathrm{mmol})$ and $3.0 \mathrm{ml}$ of pyridine $(37.2 \mathrm{mmol})$ were added to the solution. The solution was heated at $50{ }^{\circ} \mathrm{C}$ for $24 \mathrm{~h}$ with constant stirring and nitrogen purge. The mixture was then cooled to room temperature, precipitated in methanol, and dried under vacuum at $180{ }^{\circ} \mathrm{C}$ for $12 \mathrm{~h}$. The yield was $99 \%$. Polymer structures were confirmed by NMR and FTIR and are reported elsewhere [39].

\subsection{Film casting}

Polyimides were dissolved in dimethylacetamide (DMAc) to prepare a $7 \% \mathrm{wt} / \mathrm{v}(\mathrm{g}$ polymer/ml solvent) solution. Dust and particulates were removed by filtering the solution through a $0.45 \mu \mathrm{m}$ Teflon syringe filter. Afterwards, the solution was sonicated for $15 \mathrm{~min}$ to remove dissolved air. Then, the solution was cast onto a clean glass plate and initially dried under an infrared lamp at approximately $60{ }^{\circ} \mathrm{C}$ for $24 \mathrm{~h}$. The film was carefully peeled off the glass plate and soaked in deionized water to remove residual solvent. Finally, the film was heated under vacuum overnight at $210{ }^{\circ} \mathrm{C}$ for polyacetylimides and $250{ }^{\circ} \mathrm{C}$ for polyhydroxyimides. Solvent removal was confirmed using thermogravimetric analysis (TGA). 


\subsection{TR polymer conversion}

Polyimide samples were converted to polybenzoxazoles according to the reaction scheme shown in Figure 1. Although the final polybenzoxazole structure is drawn as a linear polymer, the thermal rearrangement process may also occur between neighboring polymer chains, resulting in a crosslinked structure [18]. Thermal rearrangement was performed using a Carbolite split-tube furnace (Carbolite, Watertown, WI, USA). The tube furnace was purged with nitrogen at $900 \mathrm{ml} / \mathrm{min}$. Polyimide samples were first heated at $5{ }^{\circ} \mathrm{C} / \mathrm{min}$ to $300{ }^{\circ} \mathrm{C}$ and held there for $1 \mathrm{~h}$ to ensure complete imidization. Subsequently, the samples were heated at $5{ }^{\circ} \mathrm{C} / \mathrm{min}$ to the desired rearrangement temperature (i.e., $350{ }^{\circ} \mathrm{C}, 400{ }^{\circ} \mathrm{C}$, or $450{ }^{\circ} \mathrm{C}$ ) and held at that temperature for either 30 or $60 \mathrm{~min}$. The exact thermal treatment protocol for each sample is shown in Table 3. Rearrangement conditions were chosen, based on previous studies, to minimize thermal degradation $[17,18]$. Percent TR conversion is defined as 100 times the ratio of the sample mass loss upon thermal rearrangement to the theoretical mass loss. This calculation was performed assuming that any polyimide segments that underwent thermal rearrangement were converted to polybenzoxazole segments. This approximation should be reasonable for the hydroxyl-containing samples, in the absence of thermal degradation, and its implications and limits in acetate-containing samples has been discussed previously [16, 17]. The sample mass loss was measured by weighing the sample before and after thermal treatment in the tube furnace. 


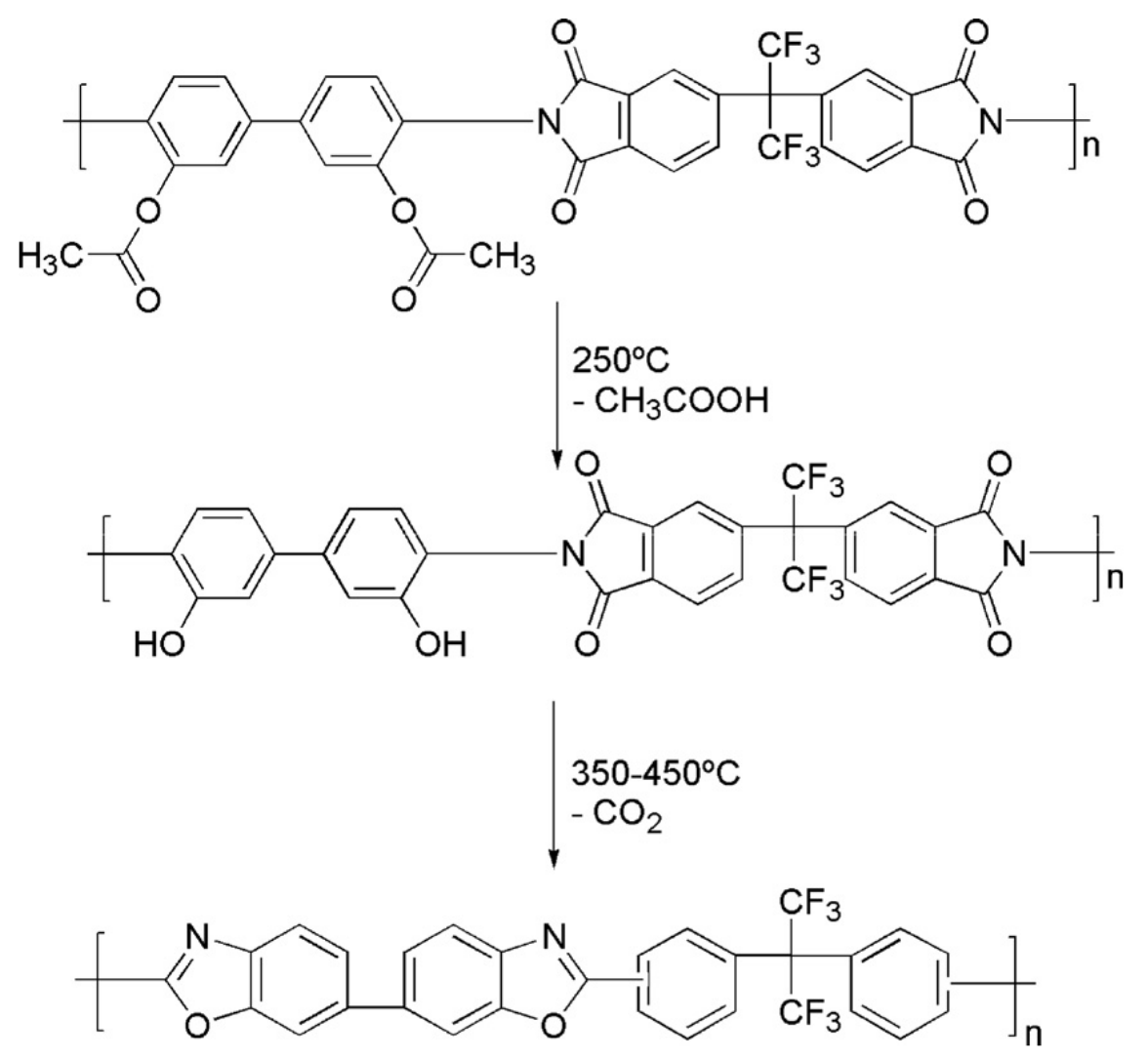

Figure 1. Thermal conversion of polyimide bearing acetate functional group (Ac sample) to the polybenzoxazole structure [18].

\subsection{Characterization}

Thermogravimetric analysis (TGA) was done using a TA Instruments Q500 Thermogravimetric Analyzer (Newcastle, DE). TGA scans were conducted at $5{ }^{\circ} \mathrm{C} /$ min under a UHP nitrogen purge ( $40 \mathrm{ml} / \mathrm{min}$ balance, $60 \mathrm{ml} / \mathrm{min}$ sample).

The glass transition temperatures $\left(\mathrm{T}_{\mathrm{g}}\right)$ of the polymers were measured using a TA Instruments Q100 differential scanning calorimeter (DSC). The samples were heated at 10 ${ }^{\circ} \mathrm{C} / \mathrm{min}$ under a $\mathrm{N}_{2}$ flow rate of $50 \mathrm{~mL} / \mathrm{min}$. The $\mathrm{T}_{\mathrm{g}}$ was calculated from the midpoint of the heat capacity step change in the first scan. 
Density was measured, based on Archimedes' principle, using a Mettler Toledo balance equipped with a density measurement kit [40]. N-heptane was used as the reference liquid because $n$-heptane has minimal interaction with the polyimides and TR polymers over the time scale of the measurement. The measurement was performed at room temperature. At least three samples were measured for each film. The average density and the experimental uncertainty are reported in Table 3.

Fractional free volume, $F F V$, was calculated as follows:

$$
F F V=\frac{V-V_{0}}{V}
$$

where $V$ is the measured specific volume $\left(\mathrm{cm}^{3} / \mathrm{g}\right)$ of the polymer sample, which is the inverse of density measured using the density kit. $V_{o}$ is the occupied volume of the polymer, and it was estimated according to the following equation:

$$
V_{0}=1.3 V_{w}
$$

where $V_{w}$ is the Van der Waals volume calculated using the group contribution method proposed by Bondi [41-43]. For partially thermally rearranged samples, occupied volume was determined using Equation (3):

$$
V_{0}=w_{T R} V_{0, T R}+\left(1-w_{T R}\right) V_{0, P I}
$$

where $w_{T R}$ is the mass fraction of the converted polybenzoxazole (PBO) structure upon thermal rearrangement, and $V_{0, T R}$ and $V_{0, P I}$ are the occupied volumes of the PBO and polyimide, respectively [18]. FFV values are presented in Table 3, and the uncertainty values were estimated based on uncertainties of density measurements using the method of propagation of errors [44]. 
Table 3. Physical and thermal properties of $m$-HAB- and $p$-HAB-based polyimides and their TR analogs.

\begin{tabular}{|c|c|c|c|c|c|c|c|}
\hline Sample & $\begin{array}{c}\text { Rearrangement } \\
\text { Temperature } \\
\left({ }^{\circ} \mathrm{C}\right)\end{array}$ & $\begin{array}{c}\text { Time at } \\
\text { Rearrangement } \\
\text { Temperature } \\
\text { (min) }\end{array}$ & $\begin{array}{c}\mathbf{T}_{\mathbf{g}} \\
\left({ }^{\circ} \mathbf{C}\right)\end{array}$ & $\begin{array}{c}\mathrm{CO}_{2} \\
\text { Theoretical } \\
\text { Mass Loss } \\
(\%) \\
\end{array}$ & $\begin{array}{l}\text { Density } \\
\left(\mathrm{g} / \mathrm{cm}^{3}\right)\end{array}$ & $\begin{array}{l}\text { Approximate } \\
\text { FFV }^{1}\end{array}$ & $\begin{array}{c}\text { \% TR } \\
\text { Conversion }\end{array}$ \\
\hline \multirow{4}{*}{$m$-HAB-6FDA } & -------- & -------- & 345 & \multirow{4}{*}{14.1} & $1.466 \pm 0.009$ & $0.141 \pm 0.007$ & -------- \\
\hline & 350 & 60 & & & $1.47 \pm 0.01$ & $0.135 \pm 0.007$ & $16 \%$ \\
\hline & 400 & 30 & & & $1.395 \pm 0.005$ & $0.167 \pm 0.003$ & $83 \%$ \\
\hline & 400 & 60 & & & $1.371 \pm 0.006$ & $0.179 \pm 0.004$ & $92 \%$ \\
\hline \multirow{4}{*}{$p$-HAB-6FDA } & ------- & ------- & 305 & \multirow{4}{*}{14.1} & $1.45 \pm 0.01$ & $0.150 \pm 0.007$ & -------- \\
\hline & 350 & 60 & & & $1.453 \pm 0.007$ & $0.145 \pm 0.005$ & $20 \%$ \\
\hline & 400 & 30 & & & $1.38 \pm 0.01$ & $0.176 \pm 0.007$ & $82 \%$ \\
\hline & 400 & 60 & & & $1.36 \pm 0.01$ & $0.187 \pm 0.007$ & $88 \%$ \\
\hline \multirow{4}{*}{$\begin{array}{c}m \text {-HAB-6FDA } \\
\text { Ac }\end{array}$} & ------- & ------- & 283 & \multirow{4}{*}{24.3} & $1.403 \pm 0.007$ & $0.161 \pm 0.006$ & -------- \\
\hline & 350 & 60 & & & $1.40 \pm 0.01$ & $0.162 \pm 0.007$ & $35 \%$ \\
\hline & 400 & 60 & & & $1.381 \pm 0.005$ & $0.173 \pm 0.003$ & $47 \%$ \\
\hline & 450 & 30 & & & $1.358 \pm 0.007$ & $0.186 \pm 0.006$ & $72 \%$ \\
\hline \multirow{4}{*}{$\begin{array}{c}p-\mathrm{HAB}-6 \mathrm{FDA} \\
\text { Ac }\end{array}$} & $\begin{array}{l}------ \\
\end{array}$ & -------- & 276 & \multirow{4}{*}{24.3} & $1.413 \pm 0.007$ & $0.155 \pm 0.005$ & ------- \\
\hline & 350 & 60 & & & $1.41 \pm 0.01$ & $0.153 \pm 0.007$ & $35 \%$ \\
\hline & 400 & 60 & & & $1.386 \pm 0.006$ & $0.170 \pm 0.004$ & $54 \%$ \\
\hline & 450 & 30 & & & $1.345 \pm 0.005$ & $0.194 \pm 0.003$ & $74 \%$ \\
\hline
\end{tabular}

${ }^{1} \mathrm{FFV}$ values were estimated based on assumption that any polyimide segments that underwent thermal rearrangement were converted to polybenzoxazole segments. A detailed analysis of the assumption's implications and limits is reported elsewhere [16]. The uncertainty values were estimated based on uncertainties of density measurements using the method of propagation of errors [44]. 


\subsection{Single gas permeation measurements}

Single gas permeability coefficients were measured using a constant-volume/variablepressure method [45]. The upstream and downstream sections of the permeability apparatus were constructed using commercially available parts from Swagelok (Austin, TX, USA). The membrane is enclosed inside a stainless steel Millipore filter holder (Millipore, Billerica, MA, USA). The permeability of $\mathrm{H}_{2}, \mathrm{CH}_{4}, \mathrm{~N}_{2}, \mathrm{O}_{2}$, and $\mathrm{CO}_{2}$ was measured in that order at $35{ }^{\circ} \mathrm{C}$ for feed pressures up to 17 atm. The gas permeability coefficient of gas $\mathrm{A}, P_{A}$, was calculated as follows [45]:

$$
P_{A}=\frac{V l}{P_{0} R T A}\left[\left(\frac{d p}{d t}\right)_{s S}-\left(\frac{d p}{d t}\right)_{l e a k}\right]
$$

where $V$ is the downstream volume, $l$ is film thickness, $P_{0}$ is the upstream pressure, $R$ is the gas

constant, $T$ is the absolute temperature, $A$ is the film area, and $\left(\frac{d p}{d t}\right)_{l e a k}$ and $\left(\frac{d p}{d t}\right)_{s s}$ are the rates of pressure rise in the downstream volume during leak test and when the gas permeation through the film is at pseudo-steady state, respectively. Permeabilities are typically reported in units of Barrer, where 1 Barrer is $10^{-10} \frac{\mathrm{cm}^{3}(\mathrm{STP}) \mathrm{cm}}{\mathrm{cm}^{2} \mathrm{~s} \mathrm{cmHg}}$. Single gas selectivity, $\alpha_{\mathrm{A} / \mathrm{B}}$, is defined as the ratio of single-gas permeabilities [45]:

$$
\alpha_{A / B}=\frac{P_{A}}{P_{B}}
$$

where $P_{A}$ is the permeability of the more permeable gas, and $P_{B}$ is the permeability of the less permeable gas. 


\section{Results and Discussion}

\subsection{TGA Characterization}

TGA scans were used to characterize the thermal rearrangement process of the polyimides. As shown in Figure 2, TGA results typically exhibit a two-stage mass loss, which is common for TR polymers $[4,12,15,17,18]$. Based on the TGA-Mass Spectroscopy analysis of hydroxyl- and acetate-containing HAB-6FDA reported by Sanders et al. [16] and Smith et al. [20], The first mass loss in the TGA scan can be attributed primarily to the thermal rearrangement process, and the second mass loss-is associated mainly with thermal degradation.

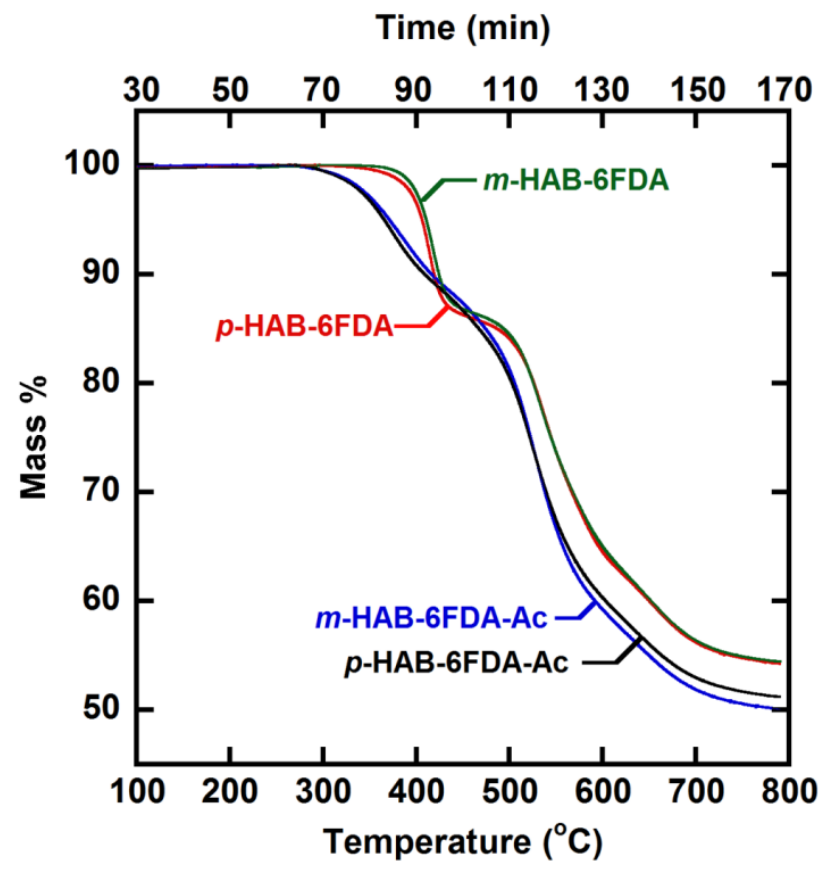

Figure 2. Thermogravimetric analysis of polymers considered in this study. Samples were heated from 50 to $800{ }^{\circ} \mathrm{C}$ at $5{ }^{\circ} \mathrm{C} / \mathrm{min}$ in a $\mathrm{N}_{2}$ atmosphere, as described in the Experimental section.

Compared with the polyhydroxyimides, the polyacetylimides exhibit a lower onset temperature of the TR process $\left(\sim 270{ }^{\circ} \mathrm{C}\right)$ and mass loss occurs over a broader temperature range. 
This gradual mass loss in the Ac samples is due to the initial conversion of acetate to hydroxyl groups before the thermal rearrangement process in these polyimides [18]. $p$-HAB-6FDA and $m$-HAB-6FDA polymers exhibit similar TGA signatures, suggesting that they undergo analogous thermal arrangement processes and have similar thermal stability.

\subsection{DSC Characterization}

Figure 3 presents first scan DSC thermograms of TR isomer polyimides. Polyhydroxyimides generally have higher $\mathrm{T}_{\mathrm{g}}$ s than polyacetylimides. For example, the $\mathrm{T}_{\mathrm{g}}$ of $p$ HAB-6FDA is $29{ }^{\circ} \mathrm{C}$ higher than that of $p$-HAB-6FDA-Ac. This difference in $\mathrm{T}_{\mathrm{g}}$ may be attributed to the fact that hydroxyl-terminated materials are generally more polar than acetatecontaining compounds [46]. For example, the dipole moment of phenol (1.72 D, measured in benzene at $\left.25^{\circ} \mathrm{C}\right)$ is $12 \%$ higher than that of phenyl acetate $(1.53 \mathrm{D}$, measured in benzene at 22 $\left.{ }^{\circ} \mathrm{C}\right)[46]$. As a result, hydroxylfunctional units are likely to have greater propensity for hydrogen bonding or other dipole-dipole interactions than acetylfunctional units, leading to higher $\mathrm{T}_{\mathrm{g}} \mathrm{s}$ for polyhydroxyimides. 


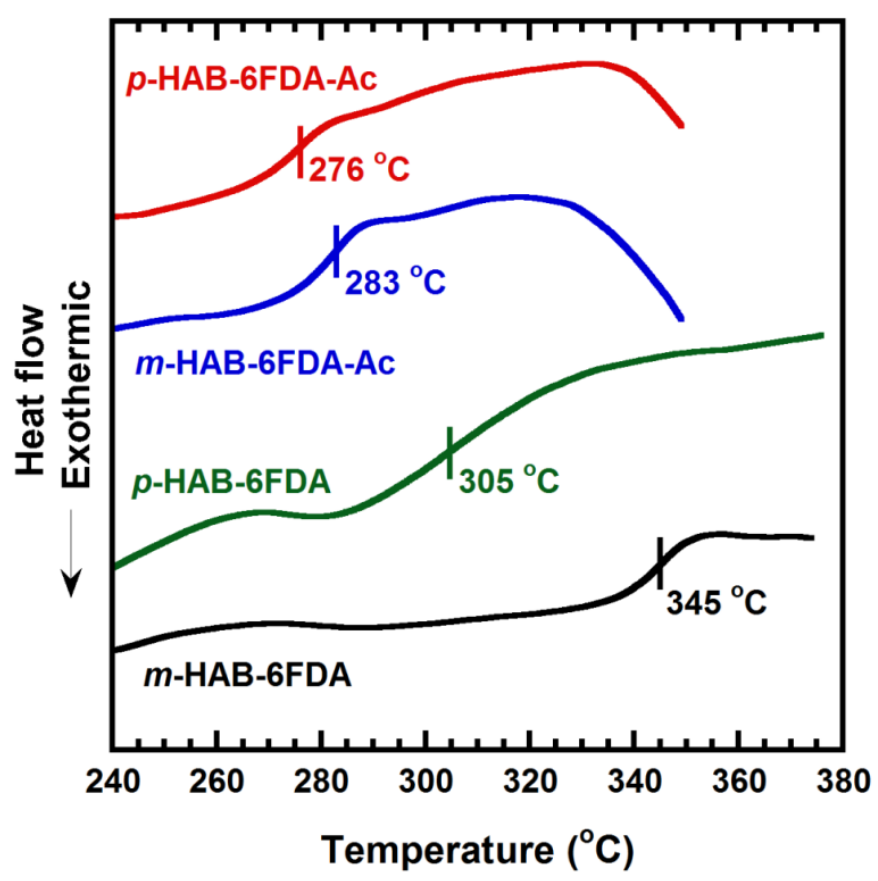

Figure 3. DSC scans of $m$-HAB- and $p$-HAB-based polyimides.

For both hydroxyl and acetate ortho-position functional groups, the $\mathrm{T}_{\mathrm{g}} \mathrm{s}$ of the metaconnected polyimides are higher than those of the para-connected analogs, although the difference in $\mathrm{T}_{\mathrm{g}}$ is greater for polyhydroxyimides $\left(\Delta \mathrm{T}_{\mathrm{g}}\right.$ of $\left.30{ }^{\circ} \mathrm{C}\right)$ than for polyacetylimides $\left(\Delta \mathrm{T}_{\mathrm{g}}\right.$ of $7{ }^{\circ} \mathrm{C}$ ). This observation is in stark contrast to the trend commonly reported for traditional nonTR aromatic polymers, where para-linked isomers typically have higher $\mathrm{T}_{\mathrm{g}} \mathrm{s}$ than meta-linked isomers [23, 27, 47] (cf., Table 1). In addition to the structural differences arising from the meta/para linkages, the polyimides considered in this study also contain either more polar (hydroxyl) or less polar (acetate) ortho-position functional groups on aromatic units that are being exchanged from para to meta configurations. In contrast, in the comparison structures shown in Table 1, typically only the connectivity of aromatic rings was changed. If the influence of these polar ortho-position functional groups, via hydrogen bonding or other dipolar interactions, is important, such interaction might compete with or even overwhelm the traditional 
meta/para effects. Additionally, as seen in previous studies, $\mathrm{T}_{\mathrm{g}} \mathrm{s}$ of polyimide precursors often overlap with the onset of thermal rearrangement $[10,16,17]$, which makes obtaining a precise value of $T_{g}$ difficult.

\subsection{Effect of ortho-position functional group on gas transport properties}

For all samples, single gas permeability coefficients of $\mathrm{H}_{2}, \mathrm{CH}_{4}, \mathrm{~N}_{2}, \mathrm{O}_{2}$, and $\mathrm{CO}_{2}$ were measured in that order at $35{ }^{\circ} \mathrm{C}$ for feed pressures ranging from 3 atm to 17 atm. Figures $4 \mathrm{~A}$ and $4 \mathrm{~B}$ show the influence of feed pressure on $\mathrm{CO}_{2}$ and $\mathrm{H}_{2}$ pure gas permeability, respectively, of $m$ HAB-6FDA-Ac TR400 60min. The permeability trends follow typical dual-mode behavior [48]. That is, for more soluble gases, such as $\mathrm{CO}_{2}$ and $\mathrm{CH}_{4}$, gas permeability decreases as feed pressure increases. For less soluble gases, such as $\mathrm{H}_{2}, \mathrm{O}_{2}$, and $\mathrm{N}_{2}$, gas permeability is essentially independent of upstream pressure [20]. Earlier studies have shown that $\mathrm{CO}_{2}$ is highly soluble in TR polymers [20], and the dual mode effect (i.e., a decrease in permeability as pressure increases) is clearly demonstrated with $\mathrm{CO}_{2}$. For example, as shown in Figure $4 \mathrm{~B}$, as feed pressure increases from 3 to 17 atm, $\mathrm{CO}_{2}$ permeability drops by $38 \%$, from 94 Barrer to 68 Barrer. Other gases, such as $\mathrm{H}_{2}$, have typically low solubility in TR polymers [20]. Therefore, dual mode effects are not apparent. As shown in Figure 4A, $\mathrm{H}_{2}$ permeability is about 166 Barrer regardless of upstream pressure. Figures of gas permeability as a function of feed pressure for other materials and other gases are included in Supplementary Information. 


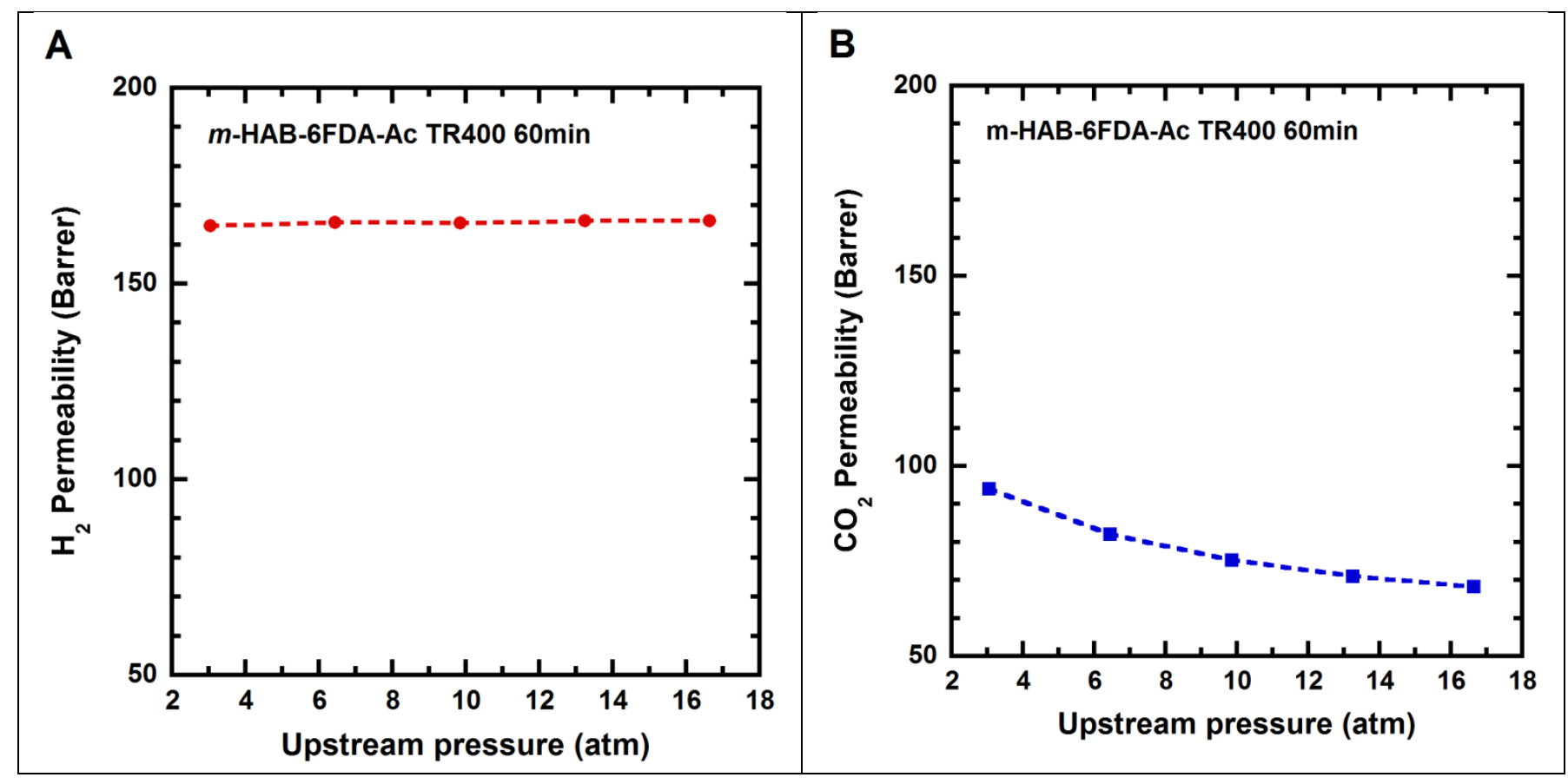

Figure 4. Effect of upstream pressure on (A) $\mathrm{H}_{2}$ and (B) $\mathrm{CO}_{2}$ permeability coefficient at $35{ }^{\circ} \mathrm{C}$ for $m$-HAB-6FDA-Ac TR400 60min.

Table 4 presents $\mathrm{CO}_{2}, \mathrm{H}_{2}, \mathrm{O}_{2}, \mathrm{~N}_{2}$, and $\mathrm{CH}_{4}$ single gas permeability coefficients in $m$-HAB and $p$-HAB-based polyimides and TR polymers. The permeability coefficients of polyacetylimides are consistently higher than those of polyhydroxyimides. For example, $\mathrm{CO}_{2}$ permeability is 5.9 Barrer for $m$-HAB-6FDA and 14.6 Barrer for $m$-HAB-6FDA-Ac. Similarly, $\mathrm{O}_{2}$ permeability is 2.1 Barrer for $p$-HAB-6FDA, compared with 4.0 Barrer for $p$-HAB-6FDA-Ac. This trend is consistent for all five gases tested for both $p$-HAB and $m$-HAB-based polyimides. Compared with polyimides with hydroxyl functionality, the polyimides with larger and less polar acetate groups can disrupt polymer chain packing more effectively and they undergo less hydrogen bonding. Both of these attributes favor higher free volume [17]. The measured density and estimated fractional free volume (FFV) values for these materials are recorded in Table 3. As shown in this table, the free volume values of polyacetylimides are higher than 
those of polyhydroxylimides, which is consistent with the higher permeabilities observed for the Ac samples.

Table 4. Single gas permeability (Barrer) of $m$-HAB- and $p$-HAB-based polyimides and TR polymers at $35^{\circ} \mathrm{C}$ and an upstream pressure of $10 \mathrm{~atm}$.

\begin{tabular}{|c|c|c|c|c|c|c|}
\hline \multirow{2}{*}{\multicolumn{2}{|c|}{ Sample* }} & \multicolumn{5}{|c|}{ Permeability (Barrer) } \\
\hline & & \multirow{2}{*}{$\begin{array}{c}\mathrm{CO}_{2} \\
5.9\end{array}$} & \multirow{2}{*}{$\begin{array}{c}\mathbf{O}_{2} \\
1.70\end{array}$} & \multirow{2}{*}{$\begin{array}{l}\mathbf{H}_{2} \\
33\end{array}$} & \multirow{2}{*}{$\frac{\mathbf{N}_{2}}{0.23}$} & \multirow{2}{*}{$\frac{\mathbf{C H}_{4}}{0.065}$} \\
\hline \multirow{4}{*}{$m$-HAB-6FDA } & PI & & & & & \\
\hline & TR350-60min & 9.0 & 2.7 & 42 & 0.40 & 0.13 \\
\hline & $\mathrm{TR} 400-30 \mathrm{~min}$ & 42 & 11.6 & 105 & 2.3 & 1.31 \\
\hline & TR400-60min & 56 & 15.0 & 124 & 3.1 & 1.80 \\
\hline \multirow{4}{*}{$p$-HAB-6FDA } & PI & 8.3 & 2.1 & 36 & 0.30 & 0.090 \\
\hline & TR350-60min & 16.3 & 4.1 & 55 & 0.65 & 0.27 \\
\hline & $\mathrm{TR} 400-30 \mathrm{~min}$ & 75 & 18.6 & 151 & 3.8 & 2.3 \\
\hline & TR400 - 60min & 73 & 18.1 & 147 & 3.7 & 2.2 \\
\hline \multirow{4}{*}{$\begin{array}{c}m-\mathrm{HAB}-6 \mathrm{FDA} \\
\mathrm{Ac}\end{array}$} & PI & 14.6 & 4.0 & 43 & 0.70 & 0.39 \\
\hline & TR350 - 60min & 16.2 & 5.1 & 67 & 0.81 & 0.30 \\
\hline & $\mathrm{TR} 400-60 \mathrm{~min}$ & 75 & 20 & 165 & 4.0 & 2.02 \\
\hline & $\mathrm{TR} 450-30 \mathrm{~min}$ & 315 & 79 & 438 & 20 & 13 \\
\hline \multirow{4}{*}{$\begin{array}{c}p-\mathrm{HAB}-6 \mathrm{FDA} \\
\mathrm{Ac}\end{array}$} & PI & 9.6 & 2.8 & 37 & 0.47 & 0.23 \\
\hline & $\mathrm{TR} 350-60 \mathrm{~min}$ & 25 & 6.8 & 80 & 1.20 & 0.50 \\
\hline & TR400 - 60min & 115 & 29 & 237 & 6.0 & 3.0 \\
\hline & $\mathrm{TR} 450-30 \mathrm{~min}$ & 443 & 114 & 582 & 30 & 21 \\
\hline
\end{tabular}

*Thermally rearranged samples are designated by the rearrangement temperature and the treatment time at that temperature.

Single gas permeability coefficients generally increase as the polyimides undergo thermal rearrangement. This trend can be seen clearly in Figure 5, which presents single $\mathrm{CO}_{2}$ permeabilities as a function of percent TR conversion for $m$-HAB and $p$-HAB-based polymers on linear (Figure 5A) and semi-log (Figure 5B) scales. For example, for $p$-HAB-6FDA-Ac, $\mathrm{CO}_{2}$ permeability increases from 9.6 Barrer in the polyacetylimide precursor to 443 Barrer for the TR450 sample. The extent of the increase in $\mathrm{CO}_{2}$ permeability also depends on the nature of the 
ortho-position functional group. TR polymers derived from polyacetylimides exhibit a larger increase in gas permeability than those derived from polyhydroxyimides. For example, $\mathrm{CO}_{2}$ permeability of $p$-HAB-6FDA-Ac TR400 60min (115 Barrer) is $58 \%$ higher than that of $p$-HAB6FDA TR400 60min (73 Barrer). Additionally, at similar TR conversions, permeability coefficients of partially rearranged polymers derived from polyacetylimides are higher than those derived from polyhydroxylimides, suggesting that bulkier ortho-position functional groups and reduced hydrogen bonding interactions may lead to TR polymers with higher gas permeability values.
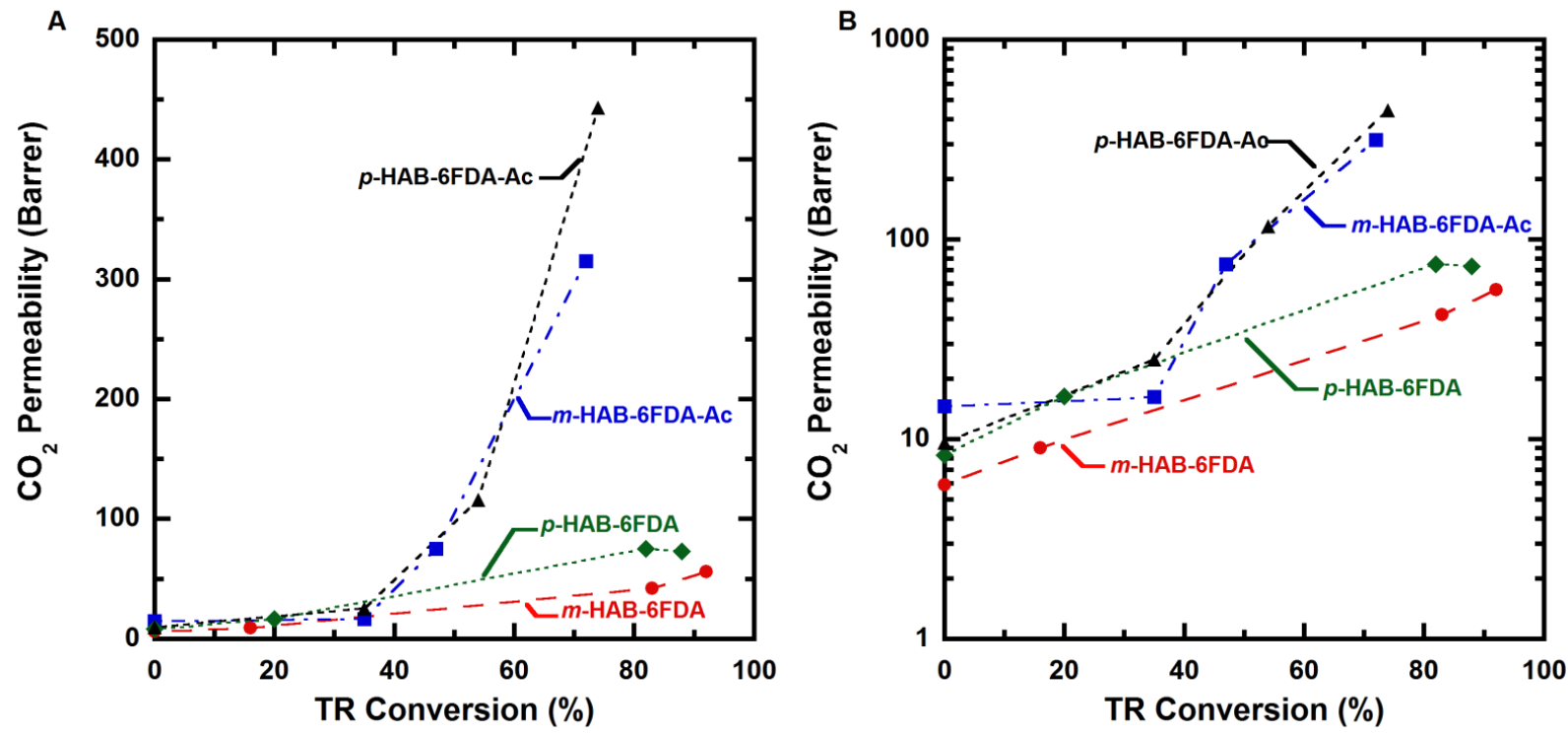

Figure 5. $\mathrm{CO}_{2}$ Permeability as a function of percent $\mathrm{TR}$ conversion on (A) linear and (B) semi$\log$ scales. Permeability values were measured at $35^{\circ} \mathrm{C}$ and an upstream pressure of $10 \mathrm{~atm}$.

Single gas selectivity values of common gas pairs are shown in Table 5. In general, gas selectivities for polyhydroxyimides are higher than those for polyacetylimides. This difference in selectivity values demonstrates a permeability/selectivity tradeoff for these materials. Figure 6 presents the single gas $\mathrm{CO}_{2} / \mathrm{CH}_{4}$ selectivity as a function of percent TR conversion for $m$-HAB 
and $p$-HAB-based samples. For the Ac samples, $\mathrm{CO}_{2} / \mathrm{CH}_{4}$ selectivity initially increased from $\sim 40$ for polyacetylimides to $\sim 50$ for TR350 samples before decreasing at higher TR conversions. As explained in previous studies, the initial increase in selectivity is presumably attributed to a more favorable free volume distribution arising from loss of the acetate group at the onset of the thermal rearrangement process $[17,20]$. For polyhydroxyimides and their TR analogs, however, the $\mathrm{CO}_{2} / \mathrm{CH}_{4}$ selectivity monotonically decreases as TR conversion increases. Polyhydroxyimides experience a much lower mass loss upon thermal rearrangement than their acetate-bearing analogs (cf., Table 3). Thus, TR samples derived from polyhydroxyimides may not have the favorable free volume distribution that occurs when Ac samples undergo the TR process. 
Table 5. Single gas selectivity of $m$-HAB- and $p$-HAB-based polyimides and TR polymers at $35^{\circ} \mathrm{C}$ and an upstream pressure of $10 \mathrm{~atm}$.

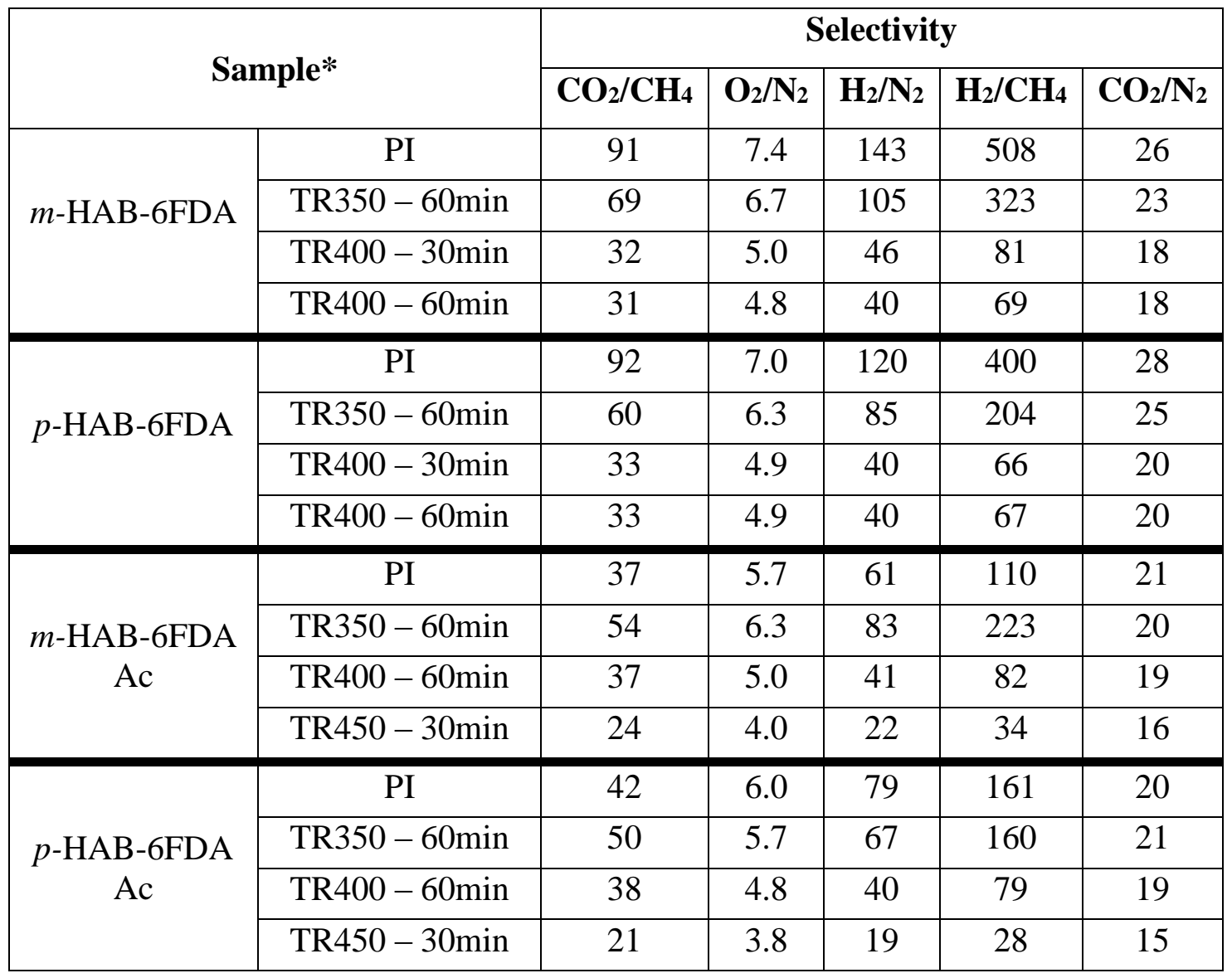

*Thermally rearranged samples are designated by the rearrangement temperature and the treatment time at that temperature. 


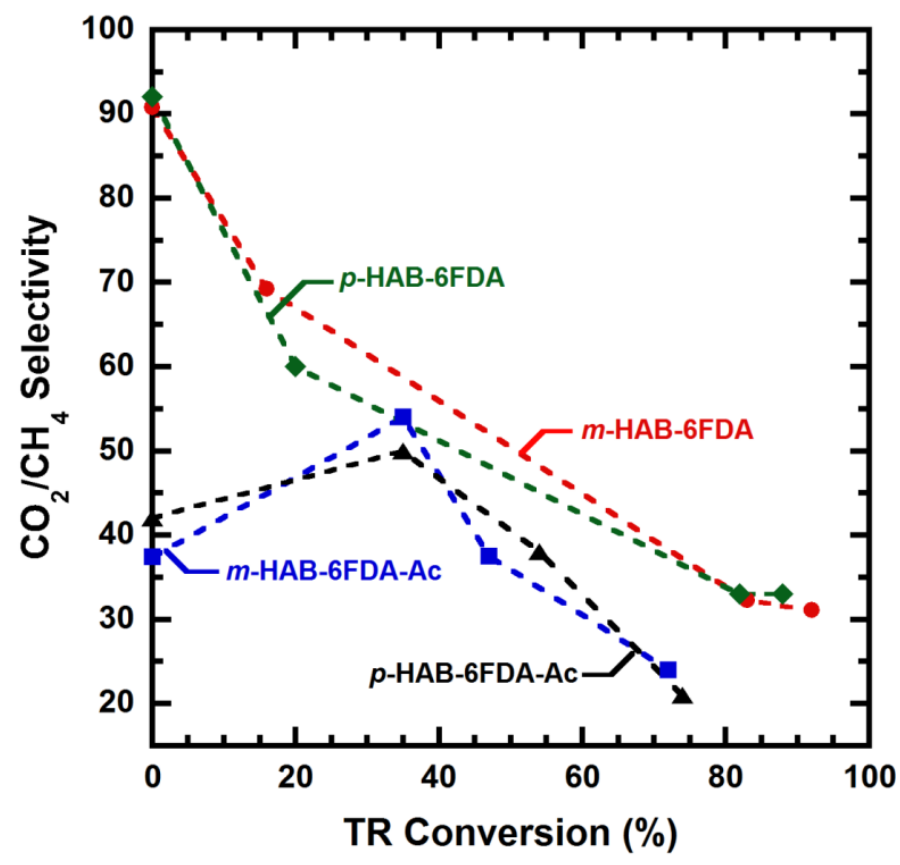

Figure 6. $\mathrm{CO}_{2} / \mathrm{CH}_{4}$ selectivity as a function of TR conversion. Permeation results were measured at $35^{\circ} \mathrm{C}$ and an upstream pressure of $10 \mathrm{~atm}$.

The monotonically decreasing trend in $\mathrm{CO}_{2} / \mathrm{CH}_{4}$ selectivity for samples with hydroxyl functionality is different from an earlier report from our group [17]. Sanders et al. reported that p-HAB-6FDA exhibited an initial rise in single $\mathrm{CO}_{2} / \mathrm{CH}_{4}$ selectivity as TR conversion increased. One likely reason for this difference is the choice of casting solvent. DMAc was used as the casting solvent for this study, whereas NMP was used by Sanders et al.. Casting solvent can have significant effects on gas transport properties. Shao et al. investigated the influence of casting solvents, such as $\mathrm{CH}_{2} \mathrm{Cl}_{2}$ and $\mathrm{NMP}$, on gas transport properties of copolyimides. A polyimide cast from $\mathrm{CH}_{2} \mathrm{Cl}_{2}\left(\mathrm{PI}-\mathrm{CH}_{2} \mathrm{Cl}_{2}\right)$ had higher gas permeability than one cast from NMP (PI-NMP) [49]. For example, $\mathrm{CO}_{2}$ permeability for $\mathrm{PI}-\mathrm{CH}_{2} \mathrm{Cl}_{2}$ was 187 Barrer, while that for PINMP was only 144 Barrer. Because $\mathrm{CH}_{2} \mathrm{Cl}_{2}$ has a lower boiling point than $\mathrm{NMP}, \mathrm{CH}_{2} \mathrm{Cl}_{2}$ can evaporate faster and may result in polymers with higher free volume [49]. In a separate study, Khulbe et al. found that physical properties, such as boiling point, viscosity, and surface tension, 
of casting solvents could all affect the gas transport properties of PPO [50]. Since, for this study, DMAc and NMP have different properties, such as the boiling point, and interactions with polyimide precursors, they may influence gas transport properties as well. In addition, films in this study were first extracted with water prior to the final high-temperature drying step during casting, whereas such an extraction step was not used in the study by Sanders et al. [10, 17]. Furthermore, strong interactions from the polar hydroxyl groups may cause physical properties of polyhydroxyimides to be rather sensitive to these differences in casting solvents and conditions. In this study, the gas permeation measurements for HAB-6FDA polyimide were repeated at least three times with different samples, and the permeability values were reproducible. The films were checked using TGA analysis to ensure complete solvent removal.

\subsection{Effect of isomer structure on gas transport properties}

The influence of meta/para linkages on gas transport properties of polyimides and their corresponding TR polymers was investigated. As presented in Table 4 and Figures 5, for polyhydroxyimides, $p$-HAB-6FDA has higher permeabilities than $m$-HAB-6FDA. For example, $\mathrm{CH}_{4}$ permeability of $p$-HAB-6FDA is $38 \%$ higher than that of $m$-HAB-6FDA. This trend is consistent with that observed in prior metalpara structure studies on linear aromatic polymers $[23,27]$. In this case, meta-connected polyhydroxyimide packs more efficiently than its paraconnected analog, as judged by the lower FFV value of $m$-HAB-6FDA presented in Table 3, which leads to lower permeability coefficients. As polyhydroxyimides undergo thermal rearrangement, the permeability coefficients of both para- and meta-connected materials increase similarly. For example, upon thermal treatment at $400{ }^{\circ} \mathrm{C}$ for $60 \mathrm{~min}, \mathrm{CO}_{2}$ permeability of $m$-HAB-6FDA and p-HAB-6FDA increases by 9.5 and 8.8 times, respectively. As presented in Table 3, $p$-HAB-6FDA has higher FFV than $m$-HAB-6FDA at every set of rearrangement 
conditions. As a result, TR polymers derived from $p$-HAB-6FDA consistently have higher gas permeability coefficients than those derived from $m$-HAB-6FDA. One exception to the permeability/FFV correlation is that the FFV of $m$-HAB-6FDA TR400 60min $(\mathrm{FFV}=0.179)$ is slightly higher than that of $p$-HAB-6FDA TR400 30min $(\mathrm{FFV}=0.176)$, even though $p$-HAB6FDA TR400 30min has higher gas permeability than m-HAB-6FDA TR400 60min. This difference may be attributed to the experimental uncertainty of density measurements and estimated FFV values (cf., Table 3).

Comesaña-Gándara et al. conducted a similar study and investigated the influence of metalpara linkages on single gas transport properties of hydroxyl-containing HAB-6FDA and its TR analogs [37]. One objective of this study is to explore whether similar metalpara effects reported by Comesaña-Gándara et al. can be replicated by studying polyhydroxyimides identical in structure but prepared using different synthesis and casting methods. Interestingly, the gas permeability coefficients of $m$ HAB 6 FDA and $p$ HАВ 6FDA polyimides and TR polymers from this study are much lower than those reported by Comesaña-Gándara et al.. For example, $\mathrm{CH}_{4}$ - permeability of $m$ HAB-6FDA from this study (0.065 Barrer) is $64 \%$ lower than the reported value (0.18 Barrer) [37]. Similarly, $\mathrm{CH}_{4}$ permeability of $p$-HAB-6FDA from this study $(0.09$ Barrer $)$ is $44 \%$ lower than what was reported $(0.16$ Barrer $)$. The differences in permeability coefficients become greater at higher TR conversions. For example, $\mathrm{N}_{z}$ permeability of $m$ HAB-6FDA TR from this study (92\% TR conversion, 3.1 Barrer) is 10 times tower than that (88\% TR conversion, 34 Barrer) reperted by Comesaña-Gándara et at. [37]. In addition to differences in permeability coefficients, the metalpara effects observed for polyhydroxyimides in this study is opposite to what was reported [37]. Comesaña-Gándara et al. observed that, contrary to the trend reported from earlier metalpara studies, the meta linked 
polyhydroxyimide has both higher permeability coefficients and higher pure-gas selectivities than its para linked analog [37]. On the other hand, as discussed earlier, the para-connected pelyhydroxyimide in this study has lower permeabilities and higher selectivities than the metaconnected isomer.

From a primary chemical structure viewpoint, the polyhydroxyimides studied by Eomesañágrndara et al. were same as the ones in this stuly. However, different synthesis routes and casting methods were employed. In the study by Comesañ-Gándara et al., HAB was first silylated before azeotropic cycloimidization to form high molecular weight polyimides [37]. In our study, polyimides were synthesized via an ester acid solution imidization. Based on previous studies, synthesis routes can have significant impacts on free volume and gas transport properties of polyimides and their TR polymers [13]. Han et al. prepared a polyhydroxyimide via either thermal or azeotropic imidization and investigated the influence of imidization route en physieal and gas transpert properties. The FFV of the polyimide prepared via thermat imidization was $12 \%$ higher than that prepared by azeotropic imidization [13]. The difference in FFV was more pronounced for partially rearranged polymers. For example, the FFV of PBO derived from thermally imidized polyimide was $27 \%$ higher than that of the PBO derived from azeotropically imidized polyimide. As a result of higher fractional free volume, the PBO derived from thermally imidized polyimide had higher gas permeability coefficients [13]. This trend of greater difference in gas permeability at higher TR conversion is consistent with that observed between our study and that reported by Comesaña-Gándara et al. [37].

In addition to differences in synthesis route, different casting solvent and casting conditions were employed. Comesaña-Gándara et al. used NMP to solution-cast the films [37], and we used DMAc as the solvent. During casting, films from this study were extracted in water 
before they were heated at high temperatures. However, Comesaña-Gándara et al. did not extract their films in water to remove residual solvent prior to the final heating step of the casting. Other casting parameters, such as solution concentration, heating rate, and drying duration, were also different. Based on previous studies, differences in casting solvent and conditions can affect physical and gas transport properties $[51,52]$. For example, $\mathrm{Bi}$ et al. reported that $\mathrm{CH}_{4}$ permeability of PTMSP cast from cyclohexane is 51\% higher than that cast from toluene and $420 \%$ higher than that cast from tetrahydrofuran (THF) [51]. These differences in permeability were consistent with changes in free volume.

Consequently, differences in synthesis and casting methods between this study and the work by Comesaña-Gándara et al. may contribute to different physical and gas transport properties reported in the two studies. For example, the fractional free volume of $m$ HAB-6FDA from this study (FFV $=0.141)$ is $17 \%$ lower than that reported by Comesaña-Gándara et al. (FFV $=0.170)[37]$, which can lead to differences in permeation properties of both polyimide precurser and its TR analogs. Moreover, Comesaña-Gándara et al. measured gas permeability at $30^{\ominus} \mathrm{C}$ and 1 atm, whereas permeability coefficients presented in Table 4 were measured at $35^{\circ} \mathrm{C}$ and 10 atm. Feed pressure can affect gas permeability according to dual mode effects, as mentioned earlier. For example, $\mathrm{CO}_{2}$ permeability of $m \mathrm{HAB}-6 \mathrm{FDA}$ measured at an upstream pressure of 3 atm is $17 \%$ higher than that measured at an upstream pressure of $10 \mathrm{~atm}$. Additionally, lower temperature typically decreases permeability due to reduced polymer segmental motion. For example, at $0 \%$ relative humidity of water, permeabilities of $\mathrm{CH}_{4}$ and $\mathrm{N}_{2}$ in Matrimid ${ }^{\circledR}-5218$ at $25^{\circ} \mathrm{C}$ are $50 \%$ and $30 \%$ lower, respectively, than those at $35^{\circ} \mathrm{C}[53]$. Therefore, with differences in synthesis routes, casting methods, and experimental conditions, a direct comparison of gas transport properties between this study and the work from Comesaña-Gándara 
et al. is difficult. Nevertheless, the observed differences in physical and transport properties demonstrate the sensitivity of these polyimide precursors to the synthesis and casting conditions and highlight the importance of adhering to a strict preparation method before making direct eomparisons of gas transport properties in these materials.

Comesaña-Gándara et al. conducted a similar study and investigated the influence of metalpara linkages on single gas transport properties of hydroxyl-containing HAB-6FDA and its TR analogs [37]. Interestingly, the gas permeability coefficients of $m$-HAB-6FDA and $p$-HAB6FDA polyimides and TR polymers from this study are substantially lower than those reported by Comesaña-Gándara et al.. However, with differences in synthesis routes, casting methods, and experimental conditions, a direct comparison of gas transport properties between this study and the work from Comesaña-Gándara et al. is difficult. A more detailed comparison and analysis between this study and that from Comesaña-Gándara et al. is included in the Supplementary Information.

Interestingly, the influence of metalpara connection on transport properties of polyacetylimides is opposite to that observed for the polyhydroxyimides. For all gases studied, $m$-HAB-6FDA-Ac has higher permeability coefficients than $p$-HAB-6FDA-Ac. For example, $\mathrm{CO}_{2}$ permeability of $m$-HAB-6FDA-Ac is $52 \%$ higher than that of $p$-HAB-6FDA-Ac. This trend is opposite to the metalpara relationships previously reported in the literature for linear aromatic polymers $[27,47,54]$. As mentioned earlier, because the rather simple para-connected materials considered previously often have more rotational mobility around the phenylene groups than their meta-connected analog, para-linked isomers typically had higher FFV, and, in turn, higher gas permeability coefficients, than that of meta-linked analogs [23, 27, 31]. However, materials from these earlier reports, some of which are presented in Table 1, typically did not have any 
substituents on the aromatic rings that are being exchanged from meta to para configurations (see highlighted aromatic units in Table 1). The presence of a bulky group, in this case the acetate group, at the ortho-position may enhance steric hindrance and dampen phenylene "ring flips" in the para isomer. As a result, acetate groups on the aromatic rings that are the site of the switch from meta to para isomers may weaken the meta/para effect observed in previous studies. These acetate groups also disrupt polymer chain packing to a greater extent for $m$-HAB-6FDAAc than for $p$-HAB-6FDA-Ac, as evident by the higher FFV in $m$-HAB-6FDA-Ac (cf., Table 3). As a result, $m$-HAB-6FDA-Ac has higher gas permeabilities than $p$-HAB-6FDA-Ac.

As polyacetylimides undergo thermal rearrangement, the acetate groups are lost, and the metalpara effect becomes more important for gas transport properties. At each thermal treatment condition, the para isomer has higher gas permeability than its meta-connected analog. For example, $\mathrm{CO}_{2}$ permeability of $p$-HAB-6FDA-Ac TR450 30min is $41 \%$ higher than that of $m$ HAB-6FDA-Ac TR450 30min. As mentioned earlier, higher free volume typically leads to higher permeability $[16-18,55]$. However, as presented in Table 3, FFVs of $p$-HAB-6FDA-Ac TR350 and TR400 samples are both lower than those of their respective meta-connected analogs. For example, FFV of $p$-HAB-6FDA-Ac TR350 is $5.6 \%$ lower than that of $m$-HAB-6FDA-Ac TR350, and FFV of $p$-HAB-6FDA-Ac TR400 is $1.7 \%$ lower than that of $m$-HAB-6FDA-Ac TR400. This apparent discrepancy in free volume values may be attributed to experimental uncertainty from density measurements. For example, experimental uncertainties of FFV for $p$ HAB-6FDA-Ac TR350 and $m$-HAB-6FDA-Ac TR350 are $4.3 \%$ and $4.6 \%$ of their respective FFV values. Another possible source of uncertainty in these FFV values is from the structural assumption of the partially rearranged polymers. FFV values of TR polymers derived from polyacetylimides were estimated based on an assumption (Assumption 1) that any polyimide 
segments remaining in the polymer following partial TR conversion maintains the acetate functionality. Another possible assumption (Assumption 2) is that polyacetylimides could be initially converted entirely to polyhydroxylimides. Then, if the sample mass loss was more than that required for the conversion of polyacetylimides to polyhydroxylimides, any further mass loss would be attributed to the conversion of polyhydroxylimides to PBOs. Sanders et al. investigated the influence of these assumptions on estimated FFV values of TR polymers derived from $p$-HAB-6FDA-Ac [16]. The authors concluded that estimated FFV values of $p$-HAB6FDA-Ac TR polymers based on Assumption 1 are 3-6\% lower than those based on Assumption 2 [16]. Finally, as mentioned earlier, loss of the acetate group at the onset of thermal rearrangement process could potentially alter free volume distribution of the polymer, which could also affect gas transport properties. If meta and para- linked polyacetylimides have different impacts on free volume distribution during thermal rearrangement, then that influence on gas permeability coefficients would not be captured in the FFV values.

In addition to the meta/para effects of polyimide precursors, there are other factors that can influence gas transport properties of TR polymers. For example, in the rearrangement of each polyimide precursor, the benzoxazole ring in the PBO can become connected to the fluorine-containing unit with either meta- or para-linkage with respect to the hexafluoroisopropylidene $[4,15]$. Figure 7 presents different possible geometric isomers of TR polymers prepared from $p$-HAB-6FDA. Statistically, three isomers are possible for each TR polymer chain, i.e., meta-meta, para-para, and meta-para (equivalent to para-meta). As a result, a mixture of meta- and para-connected PBO rings could be obtained from thermal rearrangement of either meta- or para-linked polyimide precursors. Because the exact composition of these isomeric forms in partially rearranged polymers is not known, an assessment of their respective 
impact on gas transport properties is difficult. This mixture of meta- and para-linked benzoxazole rings in PBOs also highlights the complexity in polymer structures, especially for these partially rearranged polymers, in which metalpara effects are difficult to compare. Furthermore, crosslinking of polymer chains during thermal rearrangement can also influence gas transport properties and make the metalpara effects less predictable.

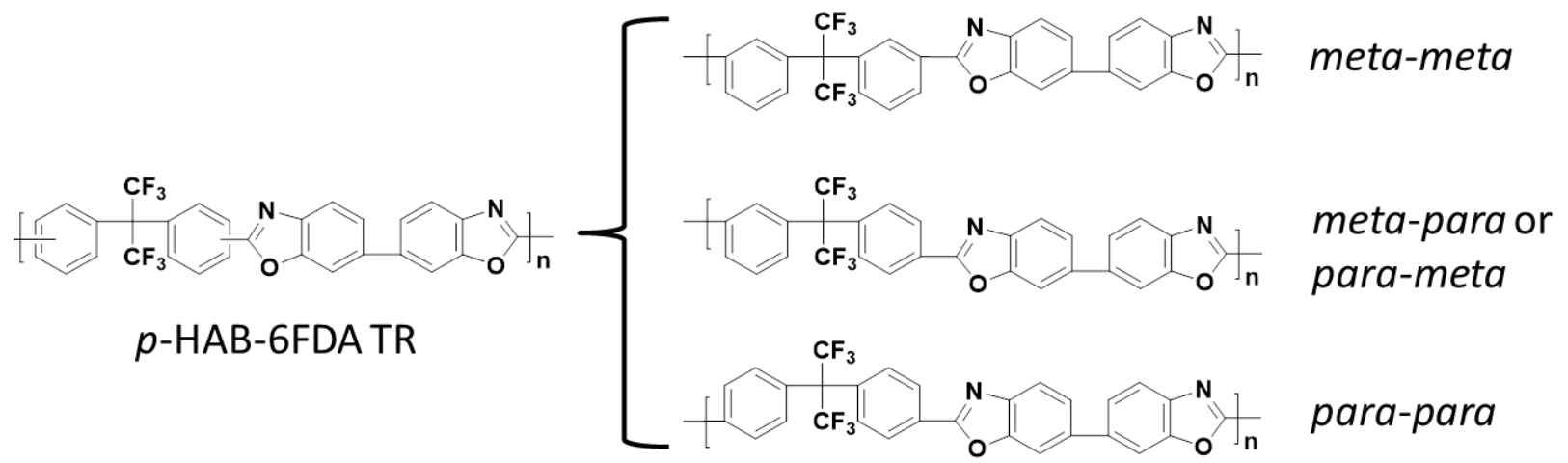

Figure 7. Possible chain conformations of TR polymers derived from $p$-HAB-6FDA.

The permeability/selectivity tradeoff for these materials is shown in Figure $8[56,57]$. In this figure, single gas $\mathrm{CO}_{2} / \mathrm{CH}_{4}$ selectivity is plotted against $\mathrm{CO}_{2}$ permeability, and percent TR conversion increases from left to right. Polyhydroxyimides exhibit $\mathrm{CO}_{2} / \mathrm{CH}_{4}$ separation properties on or near the 1991 upper bound. As these polyimides undergo thermal rearrangement, the properties move essentially parallel to the upper bound. For the acetatecontaining Ac samples, the polyacetylimides are below the upper bound, which is consistent with findings from earlier studies $[10,18,20]$. As thermal rearrangement progresses, the properties approach and eventually traverse the 1991 upper bound, indicating an improvement in $\mathrm{CO}_{2} / \mathrm{CH}_{4}$ separation performance. 


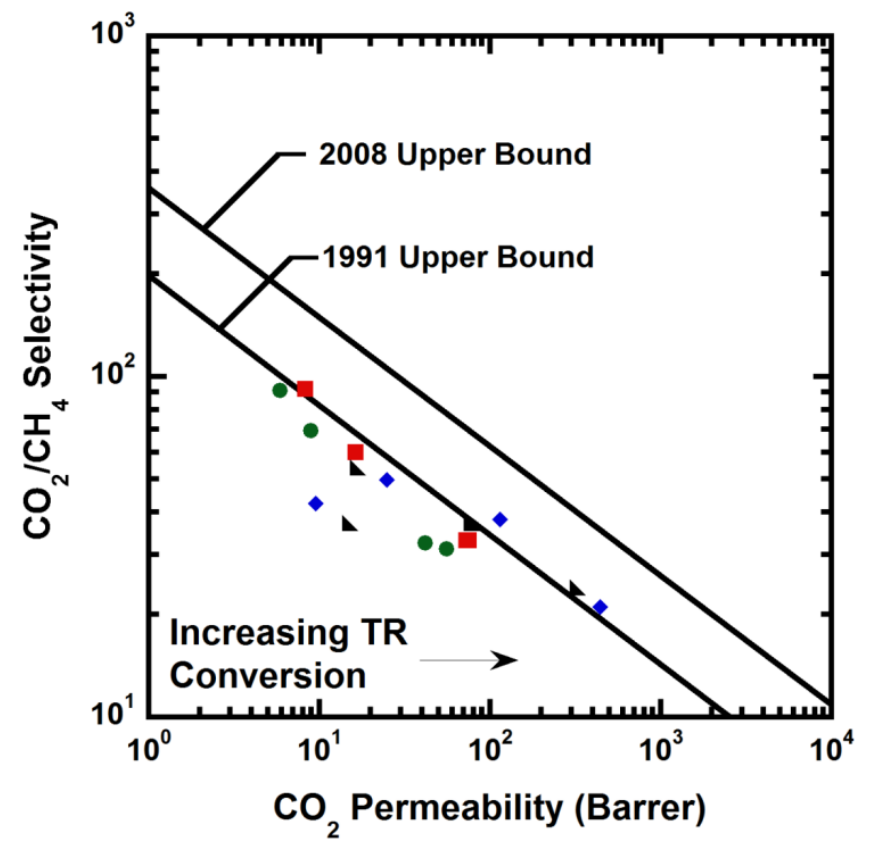

Figure 8. Upper bound plot for $m$-HAB-6FDA ( $\bullet$ ), $m$-HAB -6FDA-Ac ( $\bullet$ ), $p$-HAB-6FDA (•), and $p$ HAB-6FDA-Ac $\bullet)$. Gas transport results were measured at $35^{\circ} \mathrm{C}$ and upstream pressure of $10 \mathrm{~atm}$. Lines representing the 1991 and 2008 upper bounds are from the literature [56, 57].

Figure 9 presents single $\mathrm{CO}_{2}$ permeability as a function of reciprocal estimated fractional free volume (FFV). There appears to be a reasonable correlation between free volume and $\mathrm{CO}_{2}$ permeability. As shown in Supplementary Information, a similar correlation was also observed for other gases. This trend is consistent with earlier reports on TR polymers, which demonstrated that the increase in diffusion coefficients with increasing TR conversion was the primary contribution to the observed increase in permeability coefficients $[18,20]$. However, different polymers having similar average free volume may display rather different gas permeability coefficients. For example, $m$-HAB-6FDA TR400 60min and $m$-HAB-6FDA-Ac TR450 30min samples have almost the same free volume, but $\mathrm{CO}_{2}$ permeability of $m$-HAB6FDA-Ac TR450 30min is 4.6 times higher than that of $m$-HAB-6FDA TR400 60min. There are 
two likely reasons for this apparent discrepancy. First, as mentioned earlier, the precise chemical structure at a fixed TR conversion is not known. If the assumed composition of unconverted polyimide and polybenzoxazole does not match the actual composition, then there would be an error in the calculated occupied volume, and, in turn, the fractional free volume. Second, as mentioned in previous studies $[17,18]$, there are likely factors in addition to free volume, such as free volume distribution, that can affect the gas transport properties.

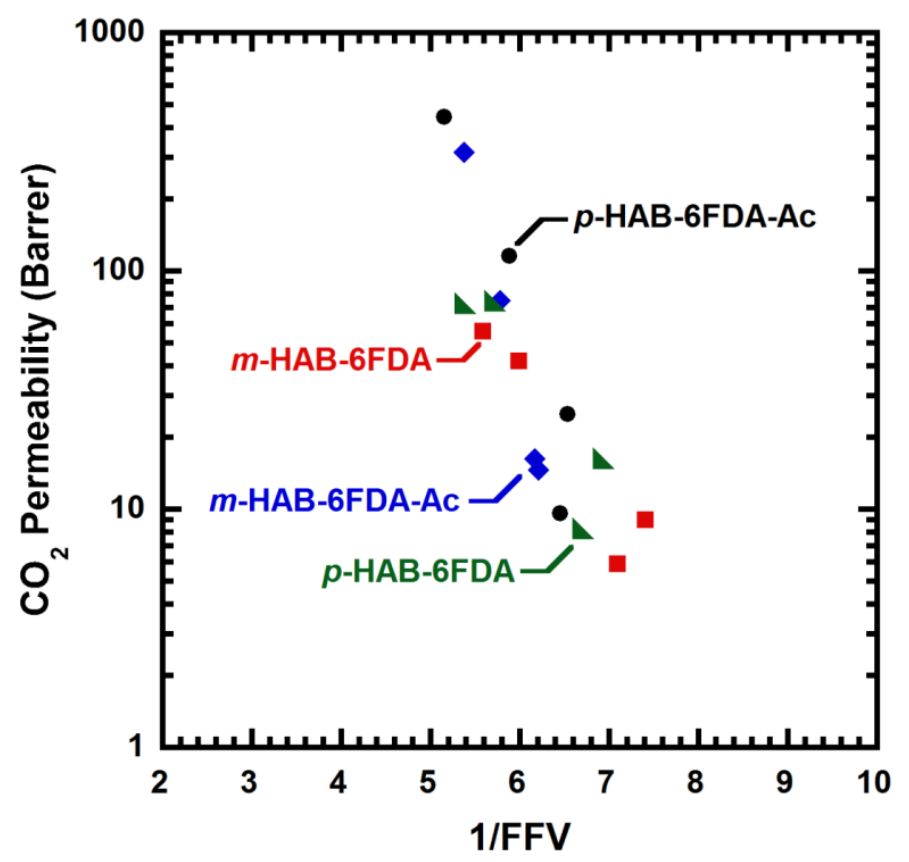

Figure 9. $\mathrm{CO}_{2}$ permeability measured at $35^{\circ} \mathrm{C}$ and an upstream pressure of 10 atm as a function of inverse free volume for $m$-HAB- and $p$-HAB-based polyimides and TR polymers.

\section{Conclusions}

Aromatic polyhydroxyimides based on HAB-6FDA were synthesized via solution imidization using an ester-acid monomer. Some of these polyhydroxyimides were acetylated to form polyacetylimides. Polyacetylimides began thermal rearrangement at a lower temperature relative to the polyhydroxyimides. The para- and meta-connected isomers showed similar 
thermal stability. Regardless of the ortho-position functionality, meta-linked polyimides had higher $\mathrm{T}_{\mathrm{gS}}$ than their para-linked analogs. The influence of ortho-position functional group structure on single gas permeability and selectivity was investigated. Polyacetylimides have higher gas permeability and lower selectivity than polyhydroxylimides. Similar results were also observed for TR polymers. Permeability values of the polyhydroxyimides in our study are lower than those reported by Comesaña-Gándara et al. [37] by about a factor of two, demonstrating that even among what are nominally the same materials, the properties could be quite different depending on the synthesis and sample preparation methods.

The influence of para vs. meta-linked structure on gas transport properties was also explored. For polyhydroxyimides and the corresponding TR polymers, $p$-HAB-6FDA had higher free volume than $m$-HAB-6FDA. As a result, the para-linked isomer had higher gas permeability than the meta-linked isomer for the polyimide precursor and TR analogs. Interestingly, the opposite trend was observed for polyacetylimides, where $m$-HAB-6FDA-Ac was more permeable than $p$-HAB-6FDA-Ac. The acetate groups may provide steric hindrance that frustrates the rotational mobility of the phenylene groups in the para-connected polyimide. As a result, the acetate group on these aromatic rings may reduce the importance of the metalpara connectivity on properties of these polymers

Finally, there was a reasonable correlation between free volume and single gas permeability. At higher TR conversions, however, TR isomers derived from either polyhydroxyimides or polyacetylimides had similar FFVs, so other factors, such as free volume distribution, may also affect gas transport properties. 


\section{Acknowledgments}

This material is based upon work supported by the U.S. Department of Energy Office of Science, Office of Basic Energy Sciences under Award Number DE-FG02-02ER15362. Financial support from the NSF Science and Technology Center for Layered Polymeric Systems (Grant No. 0423914) is gratefully acknowledged. The authors would also like to acknowledge Air Products and Chemicals, Inc. for their financial support. This study was also partially supported by the International Institute for Carbon Neutral Energy Research (WPI-I ${ }^{2} \mathrm{CNER}$ ), sponsored by the Japanese Ministry of Education, Culture, Sports, Science and Technology. 


\section{References}

[1] R.W. Baker, B.T. Low, Gas separation membrane materials: a perspective, Macromolecules, 47 (2014) 6999-7013.

[2] R.W. Baker, Future directions of membrane gas separation technology, Industrial \& Engineering Chemistry Research, 41 (2002) 1393-1411.

[3] R.W. Baker, K. Lokhandwala, Natural gas processing with membranes: an overview, Industrial \& Engineering Chemistry Research, 47 (2008) 2109-2121.

[4] H.B. Park, C.H. Jung, Y.M. Lee, A.J. Hill, S.J. Pas, S.T. Mudie, E. Van Wagner, B.D. Freeman, D.J. Cookson, Polymers with cavities tuned for fast selective transport of small molecules and ions, Science, 318 (2007) 254-258.

[5] V.J. Vasudevan, J.E. McGrath, Atomistic modeling of amorphous aromatic polybenzoxazoles, Macromolecules, 29 (1996) 637-645.

[6] G.L. Tullos, J.M. Powers, S.J. Jeskey, L.J. Mathias, Thermal conversion of hydroxycontaining imides to benzoxazoles: polymer and model compound study, Macromolecules, 32 (1999) 3598-3612.

[7] M. Calle, Y. Chan, H.J. Jo, Y.M. Lee, The relationship between the chemical structure and thermal conversion temperatures of thermally rearranged (TR) polymers, Polymer, 53 (2012) 2783-2791.

[8] M. Calle, C.M. Doherty, A.J. Hill, Y.M. Lee, Cross-linked thermally rearranged poly(benzoxazole-co-imide) membranes for gas separation, Macromolecules, 46 (2013) 81798189.

[9] J.-H. Chang, K.M. Park, S.-M. Lee, J.B. Oh, Two-step thermal conversion from poly(amic acid) to polybenzoxazole via polyimide: their thermal and mechanical properties, Journal of Polymer Science Part B: Polymer Physics, 38 (2000) 2537-2545.

[10] R. Guo, D.F. Sanders, Z.P. Smith, B.D. Freeman, D.R. Paul, J.E. McGrath, Synthesis and characterization of thermally rearranged (TR) polymers: influence of ortho-positioned functional groups of polyimide precursors on TR process and gas transport properties, Journal of Materials Chemistry A, 1 (2013) 262-272.

[11] R. Guo, D.F. Sanders, Z.P. Smith, B.D. Freeman, D.R. Paul, J.E. McGrath, Synthesis and characterization of thermally rearranged (TR) polymers: effect of glass transition temperature of aromatic poly(hydroxyimide) precursors on TR process and gas permeation properties, Journal of Materials Chemistry A, 1 (2013) 6063-6072. 
[12] S.H. Han, H.J. Kwon, K.Y. Kim, J.G. Seong, C.H. Park, S. Kim, C.M. Doherty, A.W. Thornton, A.J. Hill, A.E. Lozano, K.A. Berchtold, Y.M. Lee, Tuning microcavities in thermally rearranged polymer membranes for $\mathrm{CO}_{2}$ capture, Physical Chemistry Chemical Physics, 14 (2012) 4365-4373.

[13] S.H. Han, N. Misdan, S. Kim, C.M. Doherty, A.J. Hill, Y.M. Lee, Thermally rearranged (TR) polybenzoxazole: effects of diverse imidization routes on physical properties and gas transport behaviors, Macromolecules, 43 (2010) 7657-7667.

[14] S. Li, H.J. Jo, S.H. Han, C.H. Park, S. Kim, P.M. Budd, Y.M. Lee, Mechanically robust thermally rearranged (TR) polymer membranes with spirobisindane for gas separation, Journal of Membrane Science, 434 (2013) 137-147.

[15] H.B. Park, S.H. Han, C.H. Jung, Y.M. Lee, A.J. Hill, Thermally rearranged (TR) polymer membranes for $\mathrm{CO}_{2}$ separation, Journal of Membrane Science, 359 (2010) 11-24.

[16] D.F. Sanders, R. Guo, Z.P. Smith, Q. Liu, K.A. Stevens, J.E. McGrath, D.R. Paul, B.D. Freeman, Influence of polyimide precursor synthesis route and ortho-position functional group on thermally rearranged (TR) polymer properties: conversion and free volume, Polymer, 55 (2014) 1636-1647.

[17] D.F. Sanders, R. Guo, Z.P. Smith, K.A. Stevens, Q. Liu, J.E. McGrath, D.R. Paul, B.D. Freeman, Influence of polyimide precursor synthesis route and ortho-position functional group on thermally rearranged (TR) polymer properties: pure gas permeability and selectivity, Journal of Membrane Science, 463 (2014) 73-81.

[18] D.F. Sanders, Z.P. Smith, C.P. Ribeiro Jr, R. Guo, J.E. McGrath, D.R. Paul, B.D. Freeman, Gas permeability, diffusivity, and free volume of thermally rearranged polymers based on 3,3'dihydroxy-4,4'-diamino-biphenyl (HAB) and 2,2'-bis-(3,4-dicarboxyphenyl) hexafluoropropane dianhydride (6FDA), Journal of Membrane Science, 409-410 (2012) 232-241.

[19] C.A. Scholes, B.D. Freeman, S.E. Kentish, Water vapor permeability and competitive sorption in thermally rearranged (TR) membranes, Journal of Membrane Science, 470 (2014) 132-137.

[20] Z.P. Smith, D.F. Sanders, C.P. Ribeiro, R. Guo, B.D. Freeman, D.R. Paul, J.E. McGrath, S. Swinnea, Gas sorption and characterization of thermally rearranged polyimides based on 3,3'dihydroxy-4,4'-diamino-biphenyl (HAB) and 2,2'-bis-(3,4-dicarboxyphenyl) hexafluoropropane dianhydride (6FDA), Journal of Membrane Science, 415-416 (2012) 558-567.

[21] K.L. Gleason, Z.P. Smith, Q. Liu, D.R. Paul, B.D. Freeman, Pure- and mixed-gas permeation of $\mathrm{CO}_{2}$ and $\mathrm{CH}_{4}$ in thermally rearranged polymers based on 3,3'-dihydroxy-4,4'diamino-biphenyl (HAB) and 2,2'-bis-(3,4-dicarboxyphenyl) hexafluoropropane dianhydride (6FDA), Journal of Membrane Science, 475 (2015) 204-214. 
[22] C.A. Scholes, C.P. Ribeiro, S.E. Kentish, B.D. Freeman, Thermal rearranged poly(benzoxazole-co-imide) membranes for $\mathrm{CO}_{2}$ separation, Journal of Membrane Science, 450 (2014) 72-80.

[23] C.L. Aitken, W.J. Koros, D.R. Paul, Effect of structural symmetry on gas transport properties of polysulfones, Macromolecules, 25 (1992) 3424-3434.

[24] K. Matsumoto, P. Xu, Gas permeation of aromatic polyimides. II. Influence of chemical structure, Journal of Membrane Science, 81 (1993) 23-30.

[25] M.R. Coleman, W.J. Koros, The transport properties of polyimide isomers containing hexafluoroisopropylidene in the diamine residue, Journal of Polymer Science Part B: Polymer Physics, 32 (1994) 1915-1926.

[26] L.M. Costello, W.J. Koros, Thermally stable polyimide isomers for membrane-based gas separations at elevated temperatures, Journal of Polymer Science Part B: Polymer Physics, 33 (1995) 135-146.

[27] M.R. Coleman, W.J. Koros, Isomeric polyimides based on fluorinated dianhydrides and diamines for gas separation applications, Journal of Membrane Science, 50 (1990) 285-297.

[28] C.L. Aitken, W.J. Koros, D.R. Paul, Gas transport properties of biphenol polysulfones, Macromolecules, 25 (1992) 3651-3658.

[29] S.A. Stern, Y. Mi, H. Yamamoto, A.K.S. Clair, Structure/permeability relationships of polyimide membranes. Applications to the separation of gas mixtures, Journal of Polymer Science Part B: Polymer Physics, 27 (1989) 1887-1909.

[30] M.R. Coleman, W.J. Koros, Conditioning of fluorine-containing polyimides. 2. effect of conditioning protocol at $8 \%$ volume dilation on gas-transport properties, Macromolecules, 32 (1999) 3106-3113.

[31] R.R. Light, R.W. Seymour, Effect of sub-T $T_{g}$ relaxations on the gas transport properties of polyesters, Polymer Engineering \& Science, 22 (1982) 857-864.

[32] F.R. Sheu, R.T. Chern, Effects of packing density on the gas-transport properties of poly(phenolphthalein phthalate)s, Journal of Polymer Science Part B: Polymer Physics, 27 (1989) 1121-1133.

[33] H. Borjigin, K.A. Stevens, R. Liu, J.D. Moon, A.T. Shaver, S. Swinnea, B.D. Freeman, J.S. Riffle, J.E. McGrath, Synthesis and characterization of polybenzimidazoles derived from tetraaminodiphenylsulfone for high temperature gas separation membranes, Polymer, 71 (2015) 135-142. 
[34] S. Neyertz, D. Brown, The effect of structural isomerism on carbon dioxide sorption and plasticization at the interface of a glassy polymer membrane, Journal of Membrane Science, 460 (2014) 213-228.

[35] X.-Y. Wang, P.J. in 't Veld, Y. Lu, B.D. Freeman, I.C. Sanchez, A molecular simulation study of cavity size distributions and diffusion in para and meta isomers, Polymer, 46 (2005) 9155-9161.

[36] Y. Zhuang, J.G. Seong, Y.S. Do, H.J. Jo, M.J. Lee, G. Wang, M.D. Guiver, Y.M. Lee, Effect of isomerism on molecular packing and gas transport properties of poly(benzoxazole-coimide)s, Macromolecules, 47 (2014) 7947-7957.

[37] B. Comesaña-Gándara, M. Calle, H.J. Jo, A. Hernández, J.G. de la Campa, J. de Abajo, A.E. Lozano, Y.M. Lee, Thermally rearranged polybenzoxazoles membranes with biphenyl moieties: Monomer isomeric effect, Journal of Membrane Science, 450 (2014) 369-379.

[38] I.V. Farr, T.E. Glass, Q. Ji, J.E. McGrath, Synthesis and characterization of diaminophenylindane based polyimides via ester-acid solution imidization, High Performance Polymers, 9 (1997) 345-352.

[39] H. Borjigin, Q. Liu, W. Zhang, K. Gaines, J.S. Riffle, D.R. Paul, B.D. Freeman, J.E. McGrath, Synthesis and characterization of thermally rearranged (TR) polybenzoxazoles: Influence of isomeric structure on gas transport properties, Polymer, 75 (2015) 199-210.

[40] D. Halliday, R. Resnick, J. Walker, Fundamentals of Physics, John Wiley \& Sons, 2010.

[41] A. Bondi, van der Waals volumes and radii, The Journal of Physical Chemistry, 68 (1964) 441-451.

[42] J.Y. Park, D.R. Paul, Correlation and prediction of gas permeability in glassy polymer membrane materials via a modified free volume based group contribution method, Journal of Membrane Science, 125 (1997) 23-39.

[43] A. Bondi, Physical properties of molecular crystals, liquids, and glasses, Wiley, 1968.

[44] P.R. Bevington, D.K. Robinson, Data reduction and error analysis for the physical sciences, Third ed., McGraw-Hill, 2003.

[45] H. Lin, B.D. Freeman, Permeation and diffusion, Springer Handbook of Materials Measurement Methods, (2006) 371-387.

[46] A.L. McClellan, Tables of experimental dipole moments, W. H. Freeman, 1963. 
[47] L.A. Pessan, W.J. Koros, Isomer effects on transport properties of polyesters based on bisphenol-A, Journal of Polymer Science Part B: Polymer Physics, 31 (1993) 1245-1252.

[48] T.A. Barbari, W.J. Koros, D.R. Paul, Gas transport in polymers based on bisphenol-A, Journal of Polymer Science Part B: Polymer Physics, 26 (1988) 709-727.

[49] L. Shao, T.-S. Chung, G. Wensley, S.H. Goh, K.P. Pramoda, Casting solvent effects on morphologies, gas transport properties of a novel 6FDA/PMDA-TMMDA copolyimide membrane and its derived carbon membranes, Journal of Membrane Science, 244 (2004) 77-87.

[50] K.C. Khulbe, T. Matsuura, G. Lamarche, H.J. Kim, The morphology characterisation and performance of dense PPO membranes for gas separation, Journal of Membrane Science, 135 (1997) 211-223.

[51] J.J. Bi, C.L. Wang, Y. Kobayashi, K. Ogasawara, A. Yamasaki, Effect of the casting solvent on the free-volume characteristics and gas permeability of poly[1-(trimethylsilyl)-1-propyne] membranes, Journal of Applied Polymer Science, 87 (2003) 497-501.

[52] R. Guan, H. Dai, C. Li, J. Liu, J. Xu, Effect of casting solvent on the morphology and performance of sulfonated polyethersulfone membranes, Journal of Membrane Science, 277 (2006) 148-156.

[53] L. Ansaloni, M. Minelli, M. Giacinti Baschetti, G.C. Sarti, Effect of relative humidity and temperature on gas transport in Matrimid®: Experimental study and modeling, Journal of Membrane Science, 471 (2014) 392-401.

[54] C.L. Aitkin, D.R. Paul, Gas transport properties of polysulfones based on dihydroxynaphthalene isomers, Journal of Polymer Science Part B: Polymer Physics, 31 (1993) 1061-1065.

[55] D.F. Sanders, Z.P. Smith, R. Guo, L.M. Robeson, J.E. McGrath, D.R. Paul, B.D. Freeman, Energy-efficient polymeric gas separation membranes for a sustainable future: a review, Polymer, 54 (2013) 4729-4761.

[56] L.M. Robeson, Correlation of separation factor versus permeability for polymeric membranes, Journal of Membrane Science, 62 (1991) 165-185.

[57] L.M. Robeson, The upper bound revisited, Journal of Membrane Science, 320 (2008) 390400. 